\title{
dimensionnement des exploitations souterraines et stabilité de la surface
}

\author{
par \\ E. Tincelin \\ École des Mines, Paris \\ D. Leonet \\ D. de Winter \\ Samifer, Briey
}

CHAPITRE I : POSITION DU PROBLEME ET CRITIQUE DES SOLUTIONS ENVISAGEABLES

Le problème de la stabilité de 1a surface à 1'aplomb des exploitations minières souterraines s'est posé de tout temps :

- soit pour éviter d'endommager les édifices construits à 1'aplomb des zones exploitables en garantissant la sécurité des occupants (habitations, usines, lieux publics) ou la sécurité des utilisateurs (voies ferrées, grands axes routiers...)

- soit pour se prémunir contre les risques de venue d'eau dans les travaux souterrains en évitant de perturber 1a surface sous les voies d'eau, sous les lacs, sous la mer ou à $1^{\prime} \mathrm{aplomb}$ de terrains fortement aquifères.

- soit pour préserver des sites naturels à très grand intérêt touristique, artistique, culture1 ou écologique.

Les stots de protection sont les zones situées au fond de la mine à 1 'aplomb des surfaces du jour qu'il faut protéger. On y pratique des exploitations au taux de défruitement réduit en laissant en place suffisament de minerais pour empêcher la mise en mouvement des terrains de recouvrement.

Nous avons indiqué dans notre conférence ci-jointe intitulée "TECHNOLOGIE DES MESURES IN SITU POUR LE CONTROLE DE LA STABILITE DES STOTS ET DES CHAMBRES DE DEPILAGE"

a) les règles d'établissement des stots et les 1ois régissant les affaissements de surface,

b) les méthodes d'exploitation à 1'intérieur des stots.
En règle générale dans les mines métalliques et en particulier dans les mines de fer de Lorraine, on admet que tout mouvement de surface à 1'aplomb d'un stot doit être évité. En effet, les affaissements et les déformations du sol sont souvent brutaux et cycliques dans les mines métalliques car les édifices miniers qui les provoquent sont souvent importants et relativement proches de la surface. Ces règles et ces méthodes sont essentiellement valables dans les gisements en plateure (jusqu'à $25^{\circ}$ de pendage).

Nous allons donner ci-dessous quelques commentaires et quelques compléments à propos de ces règles et de ces méthodes d'exploitation :

\section{I-1) Réflexions sur 1e remblayage et sur ses capacités de blocage des affaissements de surface}

Les méthodes d'exploitation impliquant un défruitement total du minerai remplacé par du remblayage complet ne peuvent en aucun cas éliminer les affaissements de surface. Ainsi, si I'exploitation d'une couche de puissance $X$ mètres se fait par foudroyage (sans remblayage), on peut s'attendre à un affaissement de surface $A_{1}$ :

$$
A_{1} \cong 0,5 \mathrm{X}
$$

Si le minerai exploité est remplacé par du remblayage fait de matériaux de tout venant mis en place pneumatiquement (c'est-àdire complet et clavé au toit), on obtient un affaissement de surface $\mathrm{A}_{2}$ :

$$
A_{2} \cong 0,20 \text { à } 0,35 \mathrm{X}
$$

Si le minerai exploité est remplacé par du sable débarrassé des ultra-fins (moins de 50 microns) par cyclonage, mis en place hydrauliquement et draîné de façon permanente pendant la durée de mise en place et de tassement du remblai, on peut s'attendre à un 
affaissement de surface $\mathrm{A}_{3}$ :

$$
\mathrm{A}_{3} \cong 0,10 \text { à } 0,20 \mathrm{x}
$$

Ces données sont valables pour les mines métalliques et ont été vérifiées jusqu'à 400 mètres de profondeur. Dans les houillères, généralement plus profondes, on trouve (moyenne des mesures européennes) :

$$
\begin{aligned}
& A_{1} \cong 0,9 \mathrm{x} \\
& \mathrm{A}_{2} \cong 0,3 \text { à } 0,4 \mathrm{x} \\
& \mathrm{A}_{3} \cong 0,15 \text { à } 0,2 \mathrm{x}
\end{aligned}
$$

On voit donc que 1 e remblayage, que 1 qu'il soit, ne peut procurer la solution parfaite du problème posé à savoir 1'élimination des mouvements de surface sauf en y adjoignant du ciment (béton maigre). Le remblayage classique ne peut que diminuer notablement 1 'intensité des mouvements de surface. Par contre, le remblayage peut renforcer des piliers abandonnés en les confinant et en les préservant des effets de $1^{\prime}$ altération.

Disons cependant, que de très nombreux minerais à faible valorisation ne peuvent pas supporter 1a charge financière d'exploitation résultant de 1 a mise en place correcte du remblayage total ou partiel à fortiori lorsqu'il est cimenté. C'est le cas en France des mines de fer et des mines de bauxite entre autres.

En d'autres termes, il faut mettre au point d'autres solutions ne mettant pas en oeuvre le remblayage même partiel.

\section{I-2) Les piliers abandonnés}

Les piliers abandonnés (fig. $n^{\circ} 1$ ) peuvent constituer une solution au problème posé lorsque le taux de défruitement a été correctement établi en tenant compte des résistances mécaniques des bancs exploités, des bancs immédiats du toit et du mur.

On adopte comme critère de rupture celui déduit des limites élastiques diminuées d'un écart-type. Un calcul par éléments finis s'impose souvent. En première approximation, on peut se contenter d'un calcul simplifié à condition de se fixer par exemple la largeur des galeries $l$ ceinturant les piliers et la géométrie de ces derniers, par exemple: piliers carrés de $\mathrm{x}$ mètres de côté :

$$
\mathrm{R} \leqslant \rho \mathrm{g} H \frac{1}{1-\tau}
$$

$\tau=$ taux de défruitement $=\frac{l(l+2 x)}{(x+l)^{2}}$

$\mathrm{H}=$ profondeur

$\varphi g=$ poids spécifique des roches du recouvrement

$\mathrm{R}=$ résistance en compression monoaxiale

$=R_{E}-\varepsilon$

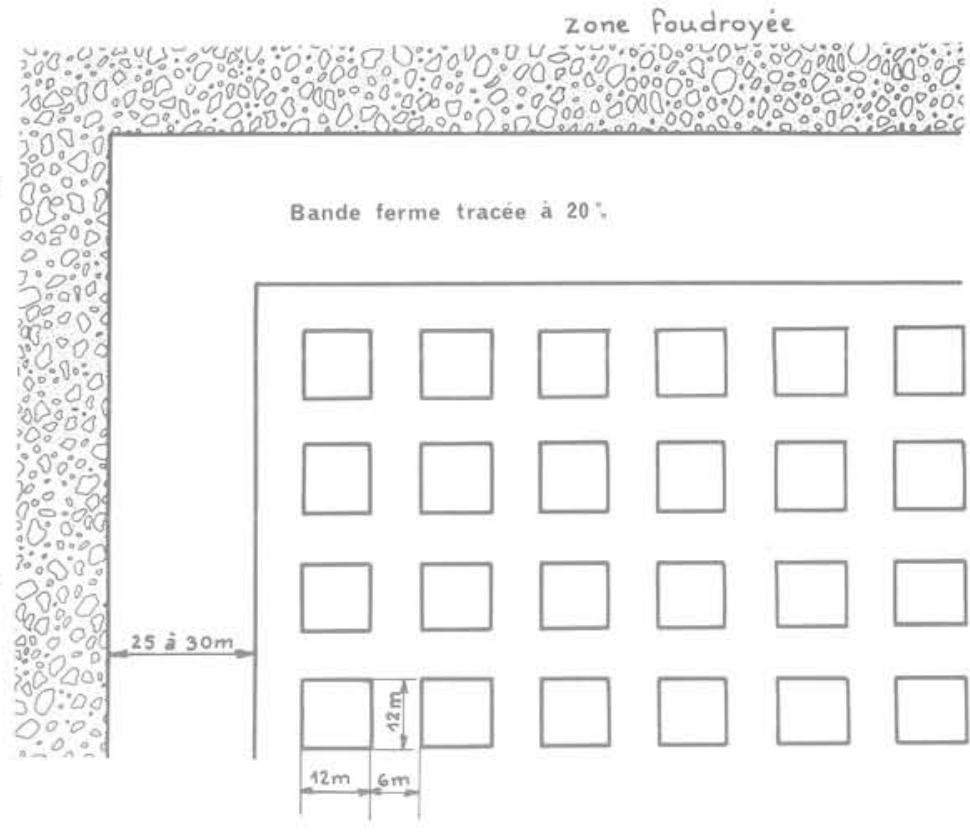

Fig.l Piliers abandonnés

avec $R_{E}=1$ imite élastique en compression monoaxiale

$\varepsilon=$ écart-type

Prenons l'exemple d'une mine de fer de Lorraine située à une profondeur $\mathrm{H}$ avec : $\mathrm{R}=9.10^{6}$ Pascal et $\varphi \mathrm{g}=25000$ Newton $/ \mathrm{m}^{3}$

$\begin{array}{ll}\text { Si H }=220 \mathrm{~m} & \tau \cong 39 \% \\ \text { Si H }=180 \mathrm{~m} & \tau \cong 50 \% \\ \text { Si H }=140 \mathrm{~m} & \tau \cong 61 \%\end{array}$

Admettons des piliers abandonnés de for-. me carrée de $x$ mètres de côté et une profondeur $\mathrm{H}=180$ Mètres.

On obtient $x \cong 12$ mètres si $=6$ mètres

On obtient $x \cong 10$ mètres si $=5$ mètres

Ce calcul simpliste qui ne tient pas compte de nombreux facteurs en particulier de la hauteur des piliers donne cependant une idée approximative du taux recherché. On voit de toute manière que très rapidement en fonction de la profondeur, on ne peut plus obtenir de taux de défruitement satisfaisant. (La formule simplifiée est de moins en moins valable au fur et à mesure que les piliers deviennent moins élancés.)

Les calculs par éléments finis sont certes plus précis et plus complets puisqu'ils renseignent en plus sur la tenue des toits des galeries ceinturant les piliers. Mais ces calculs comportent une certains approximation car à 1 'heure actuelle, ils restent à deux dimensions, ce qui impose de remplacer les petits piliers abandonnés par le pilier long équivalent et de plus, ils ne tiennent pas compte de 1 a fissuration naturelle. 
Certes, on peut par le raisonnement modifier les résultats pour tenir compte de cette fissuration nature11e, surtout lorsqu'elle est peu complexe. Mais il reste encore une autre indétermination : nous voulons calculer des édifices pour une durée de vie illimitée. Or, les caractéristiques mécaniques de certaines roches peuvent subir en fonction du temps des altérations qui peuvent entraîner à terme des instabilités des piliers abandonnés.

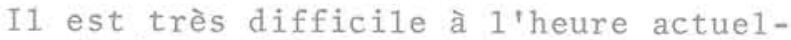
le de prévoir et de tenir compte dans les calculs de ces effets de l'altération de certaines roches en fonction du temps engendrée par 1'action de $1^{\prime}$ air ou de $1^{\prime}$ eau ou par 1'action de mises en charge permanente (fatigue).

Malgré les précautions prises et malgré le respect de certaines règles, on a constaté $1^{\prime}$ effondrement à terme de zones exploitées par petits piliers qui pourtant avaient "tenu" de nombreuses années (p1us de 50 ans et même plus d'un siècle). Par ailleurs, il est bon de rappeler que les piliers abandonnés établis à la suite d'une erreur d'exploitation avec un taux de défruitement trop important peuvent conduire à des incidents très graves résultant des effondrements spontanés.

I1 est donc normal que $1^{\prime}$ on ait cherché à remplacer les piliers abandonnés par une autre méthode d'exploitation, en particulier lorsque $1^{\prime}$ on craignait les effets de I'altération ou de la fatigue en fonction du temps. Enfin, ne pouvait-on pas obtenir avec une autre méthode d'exploitation des structures souterraines stables mais procurant des taux de defruitement plus importants qu'avec les piliers abandonnés ? Ce dernier aspect devient d'autant plus important que le gisement concerné s'approfondit.

\section{I-3) Les îlots classiques et les îlots réduits}

Dans les mines de fer de Lorraine, plus spécialement dans celles où 1 a résistance des roches est faible ou dans celles où la présence de marnes entraîne ou peut entraîner des altérations en fonction du temps, on a pensé depuis plus de 70 ans à remplacer les piliers abandonnés par la méthode dite des llots (fig, $\mathrm{n}^{\circ}$ ).

Celle-ci consiste à exploiter complètement par foudroyage des bandes de largeur limitée à L. A 1'aplomb de cette zone exploitée et foudroyée, on obtient la formation d'une "cloche" de roches foudroyées et fracturées. Si la largeur L est bien estimée on peut espérer ne pas atteindre, ni tangenter la surface du jour avec le sommet de la cloche.

Entre les bandes ainsi exploitées, on

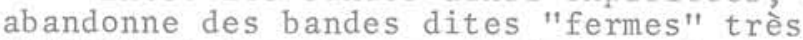
faiblement tracées par quelques galeries.

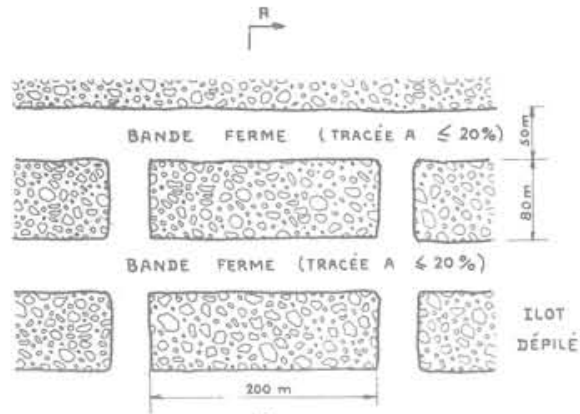

$\stackrel{\mathrm{B}}{\longrightarrow}$

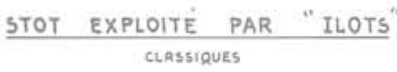

COUPE $A \cdot B$

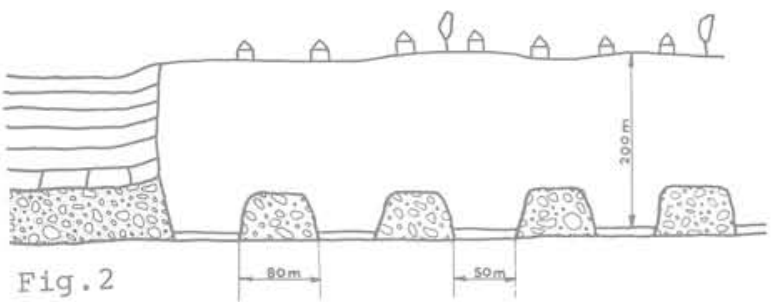

Ces bandes fermes doivent avoir une largeur $\lambda$ suffisante pour soutenir $1^{\prime}$ ensemble des terrains du recouvrement.

Le problème consiste donc à déterminer $L$ et $\lambda$. Cette détermination s'est effectuée de manière empirique en analysant dans les mines de fer les îlots $n^{\dagger}$ ayant entrấné aucun affaissement de surface sur de nombreuses années (plus de 50 ans).

$$
\text { On a trouvé : } \begin{aligned}
& \mathrm{L} \\
& \quad \lambda \geqslant 0,4 \mathrm{H} \\
& \lambda \geqslant 0,2 \mathrm{H}+12
\end{aligned}
$$

Ces formules totalement empiriques ont été vérifiées pour des valeurs de $\mathrm{H}$ comprises entre 230 mètres et 120 mètres dans 1 es seules mines de fer de Lorraine.

Le taux d'ilotage devient, en supposant que l'exploitant ait pu exploiter totalement 1 a bande exploitée et foudroyée :

$$
\tau=\frac{L}{L+\lambda}=\frac{0,4 H}{0,6 H+12}
$$

Cette formule empirique est assez criticable puisqu'elle ne tient pas compte des caractéristiques mécaniques des roches concernées et puisqu'elle conduit à un taux d'ilotage qui augmente 1 égèrement avec $1 \mathrm{a}$ profondeur :

$$
\begin{aligned}
& \tau=55 \% \text { pour } H=100 \text { mètres } \\
& \tau=61 \% \text { pour } H=230 \text { mètres }
\end{aligned}
$$

Une explication à ces résultats apparemment surprenants pourrait être $1^{\prime}$ accroissement de résistance des bandes fermes dont la résistance varierait plus vite que leur largeur ou plus simplement résulterait d'une erreur dûe à la faiblesse du nombre des résultats pratiques pris en compte pour l'établissement de ces formules dans un domaine de variation assez étroit de la hauteur du recouvrement. 
Disons que jusqu'à ce jour, aucun stot de protection établi en respect de ces règles de géométrie, qui de plus limitent strictement la surface des quelques galeries creusées dans les bandes fermes (moins de $20 \%$ ) ne s'est effondré ou n'a manifesté de signes de fatigue. Les affaissements de surface relevés sont toujours restés nuls. Les taux de défruitement obtenus sont supérieurs à ceux résultant des piliers abandonnés.

Par contre, cette façon d'opérer satisfaisante pour la stabilitê de 1 a surface et pour le taux de défruitement procurait et procure deux inconvénients majeurs :

a) Ia hauteur des cloches obtenue avec $\mathrm{L}=0,4 \mathrm{H}$ est inconnue mais très souvent suffisante pour entrainer la rupture des bancs imperméables qui forment un écran protecteur particulièrement efficace vis$a-v i s$ des venues d'eau au fond. Dans les mines de fer, il s'agit des marnes micacées (fig. n ${ }^{8} 3$ ). Or, la présence de l'eau dans les travaux du fond entrâne d'importants frais d'exhaure (grande consommation d'électricité) et des contitions d'exploitation pénibles pour $1 \mathrm{e}$ personnel et pour le matériel.

b) $1 \mathrm{a}$ 1argeur $\mathrm{L}=0,4 \mathrm{H}$ est assez grande pour entraîner pendant 1'exploitation par dépilage et foudroyage des bandes exploitées L, des difficultés comparables à celles qui surviennent dans les fronts de dépilage de grandes dimensions (supérieure à L). En particulier, les pressions de terrain $y$ sont aussi fortes.

Pour éviter ces deux inconvénients (présence de l'eau dans les travaux du fond et conditions de pression importantes), il a été décidé de tenter une modification de la méthode d'exploitation par îlots en réduisant les dimensions $L$ et $\lambda$. Cette réduction devait entrainer une diminution de $1 \mathrm{a}$ hauteur des cloches de foudroyage. On espérait ainsi ne plus affecter les marnes micacées, c'està-dire ne plus détruire le "parapluié" du mineur. La hauteur des terrains mis en mouvement par 1'exploitation ayant été réduite, on pouvait escompter une diminution des pressions imposées aux travaux d'exploitation entrepris à 1'intérieur des bandes exploitées.

Cette réduction des largeurs $\mathrm{L}$ et $\lambda$ a donné 1e nom d' "ILOTS REDUITS" à cette variante de l'exploitation des stots de protection par îlots.

Pour 1a mise au point des ILOTS REDUITS, nous avons pour la première fois associé deux techniques menées simultanément :

a) des essais en vraie grandeur entrepris au fond de plusieurs mines en mettant en oeuvre plusieurs campagne de mesures (mesures des affaissements de surface - des déformations subies par les bandes fermes séparant les îlots - des hauteurs de "cloches"). Les mesures en question devaient se faire souvent à distance puisqu'il n'y
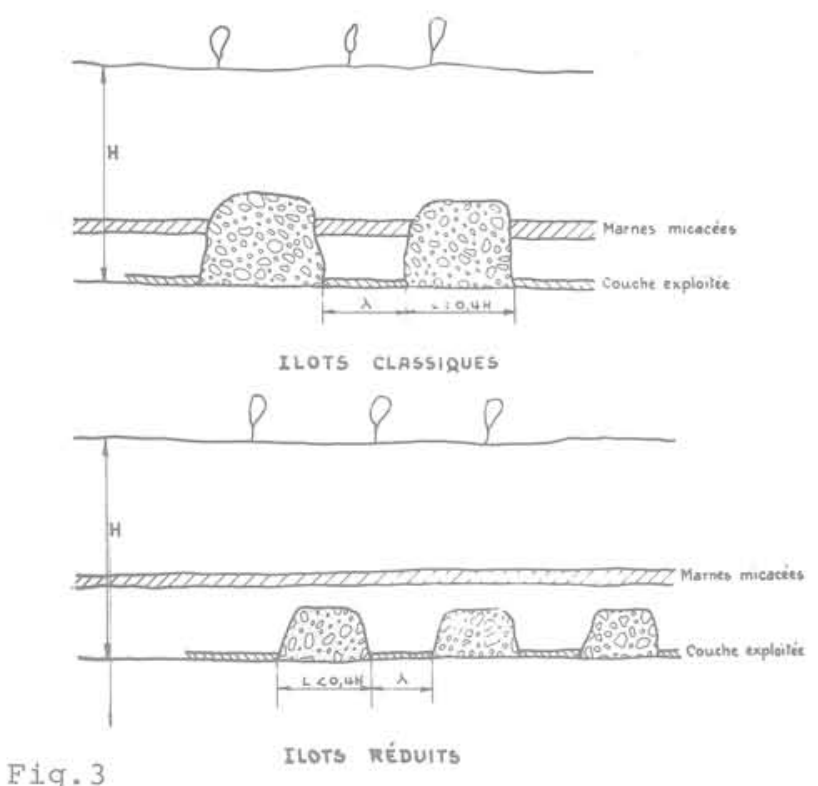

avait plus accès aux bandes fermes après 1'exploitation des îlots contigüs.

b) mise au point de méthodes de calcul permettant de prévoir 1a hauteur des cloches en fonction de $L$ ainsi que les largeurs $\lambda$ des bandes fermes en fonction de $L$.

La confrontation des essais pratiques,
des mesures et contrô-
les in situ
des calculs prévision-
nels, a permis la mise au point relativement
de de cette nouvelle technique minière.

La note ci-jointe a pour but de reprendre l'ensemble de ces essais en montrant 1 a part primordiale prise par les techniques de la mécanique des roches dans $1^{\prime}$ obtention de ces résultats.

\section{I-4) Extension du domaine d'emploi des îlots réduits}

La technique des îlots réduits évitant Ia venue de $I^{\prime}$ eau au fond et limitant considérablement les pressions dans les travaux d'exploitation constitue, en dehors des stots une méthode d'exploitation efficace lorsque les roches à exploiter sont trop peu résistantes pour subir les contraintes nées par exemple lors d'un dépilage classique. La figure $\mathrm{n}^{\circ} 4$ rappe11e 1 a géométrie et 1 e principe d'exploitation du dêpilage classique (chambres et piliers avec dépilage). Dans cette façon d'exploiter, on réussit normalement à obtenir un taux de défruitement voisin de $85 \%$ et même de $90 \%$. Dans les mines difficiles, le dépilage classique entrâne des abandons importants de minerai consécutifs aux mises en charge permanentes ou cycliques. Il n'est pas rare de ne pouvoir défruiter son gisement à plus de 50 ou $60 \%$, contraint et forcé par les événements. Ces abandons se font par ailleurs dans un contex- 


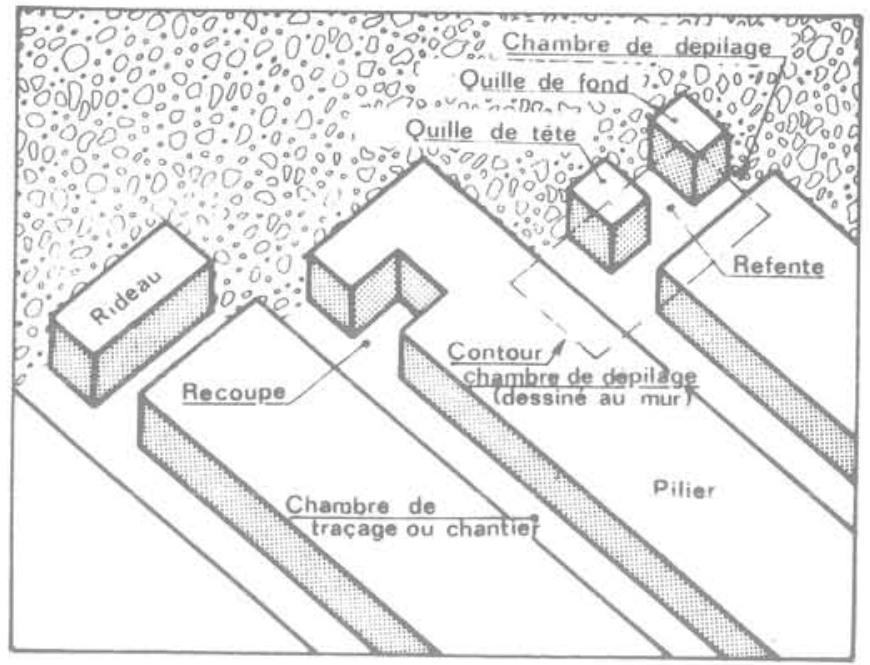

Fig.4 Schéma de principe de l'exploitation par chambres et piliers avec dépilage et foudroyage sur quilles résiduelles te relativement dangereux et très coâteux de travaux difficiles et exceptionnels entrepris à la demande et avec des travaux considérables de renforcement. Les îlots réduits conduisent sensiblement au même taux de défruitement de $60 \%$ et ils aboutissent à des conditions d'exploitation bien plus systématiques, plus sereines, sans danger pour le personnel et en outre moins couteuses.

La methode d'exploitation dite des "îlots réduits" n'est donc pas seulement réservée aux stots de protection mais elle tend de plus en plus à être utilisée à la place des méthodes d'exploitation classiques quand celles-ci deviennent inapplicables. La mise au point de cette méthode d'exploitation et la détermination des largeurs des ilots et des bandes fermes s'impose donc impérativement.

$C^{\prime}$ est le problème que nous avions à résoudre. Nous exposons ci-dessous le déroulement de nos études et les résultats actuels obtenus.

\section{CHAPITRF II : L'ETUDE DU DIMENSIONNEMENT DES ILOTS REDUITS} II-1) Résultats des essais entrepris in situ

I I-1-1) Mine_de Joudrevi11e

A) Le recouvrement et 1a détermination empirique des_dimensions des î́n réduit

La mine de fer de Joudreville exploite 1a couche $\mathrm{L}_{1}$, dite couche noire (minerai siliceux) de $3,4 \mathrm{~m}$ de puissance située à une profondeur variant entre 200 et 220 mètres.

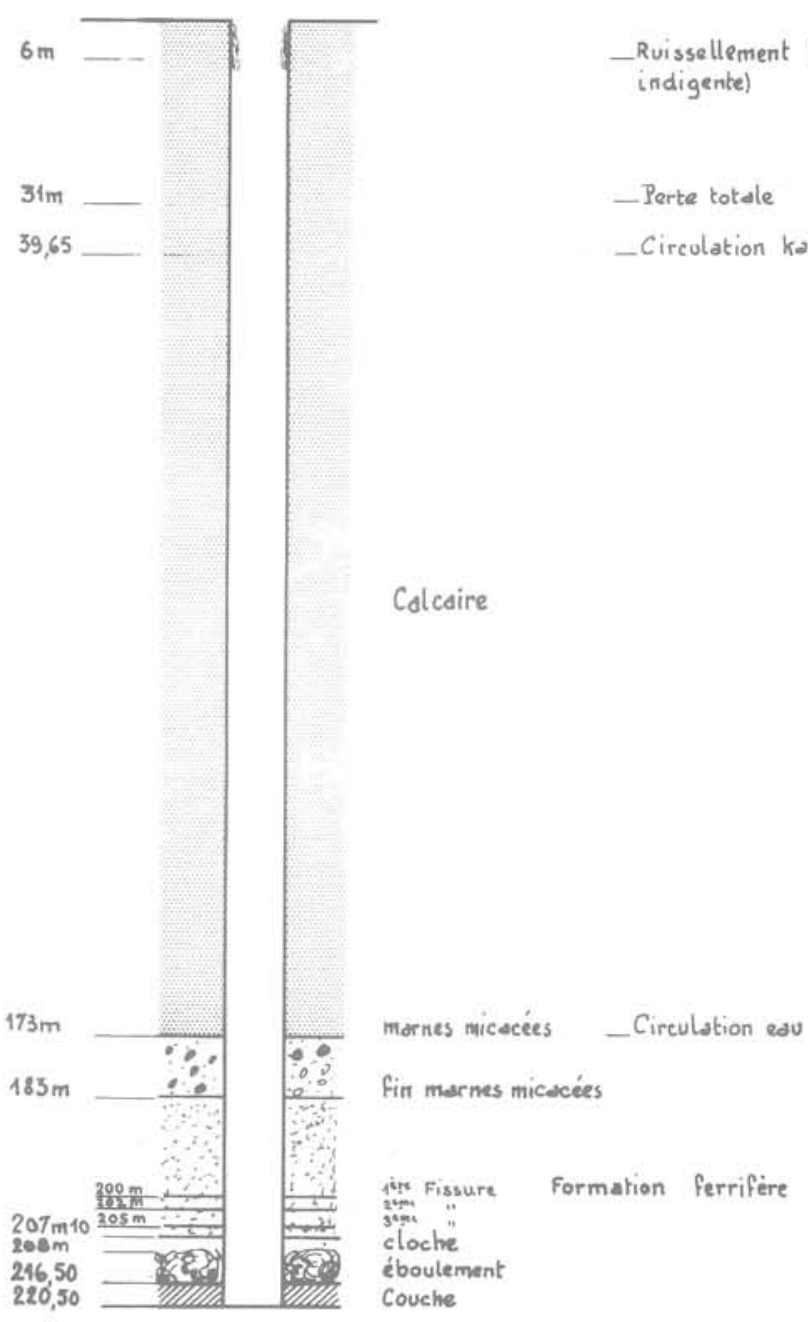

Fig.5 Coupe sondage Joudreville

Le recouvrement (fig, $\mathrm{n}^{\circ} 5$ ) comporte en partant du mur de la couche situé dans 1e sondage pris comme référence à $220,5 \mathrm{~m}$ de profondeur (comptée à partir de 1'orifice du sondage) :

a) 1a formation ferrifère dont le sommet se situe à une profondeur de $183 \mathrm{~m}$. Cette formation ferrifère de $37,5 \mathrm{~m}$ d'épaisseur est constituée d'une alternance de bancs de marnes et de couches minéralisées dont 1a plus importante, 1 a couche dite grise de $5 \mathrm{~m}$ de puissance, a son mur situé à $12 \mathrm{~m}$ du mur de 1 a couche noire exploitée. La formation ferrifère est située dans 1'AALENIEN.

b) Ies marnes micacées de $10 \mathrm{~m}$ d'épaisseur ont leur mur situé à une profondeur de $183 \mathrm{~m}$. Elles font partie également de 1'AALENIEN.

c) au-dessus des marnes micacées, débutent les bancs calcaires du BAJOCIEN inférieur puis du BAJOCIEN supérieur, surmonté des bancs du BATHONIEN. Ces bancs souvent épais et naturellement fissurés sont constitués de roches dures et assez dures. Les bancs calcaires les plus proches des marnes micacées bien que durs et très durs, 
sont barrés par les circulations d'eau karstique.

$$
\circ \circ
$$

Le but recherché par les essais était de constituer des illots réduits dont les cloches de foudroyage ne montaient pas à $183 \mathrm{~m}$ de profondeur pour ne pas atteindre les marnes micacées qui constituent le parapluie du mineur.

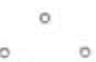

Les formules empiriques des ilots classiques appliquées au cas de Joudreville autorisaient la constitution d'îlots classiques de largeur :

$$
\mathrm{L}=0,4 \mathrm{H}=0,4 \times 220=88 \mathrm{~m}
$$

Ces ilots auraient eu leur sommet dans les bancs durs et très durs des calcaires surmontant les marnes micacées soit à 50 ou 60 mètres de hauteur. Pour rester à une hauteur inférieure à 1 a moitié de cette hauteur de $60 \mathrm{~m}$, soit 25 à $30 \mathrm{~m}$ afin de ne pas atteindre les marnes micacées, nous avons pensé qu'il était nécessaire de limiter 1a largeur L pour les îlots réduits à une valeur légèrement inférieure à la moitié de la largeur maximale $\mathrm{L}=0,4 \mathrm{H}$.

Nous avons adopté pour cet essai d'îlots réduits une largeur :

\section{$\mathrm{L}=38$ mètres}

avec un taux d'ilotage voisin de $60 \%$ identique à celui des îlots classiques de grandes dimensions. On obtenait donc :

$$
\frac{38}{38+\lambda}=60 \%
$$

soit $\lambda \cong 26$ mètres.

On obtenait donc un schéma d'exploitation tel que celui décrit dans la figure 6.

Les bandes fermes ayant une largeur $\lambda$ de $26 \mathrm{~m}$ ont été percées de quelques galeries (uniquement transversales) nécessaires à l'exploitation et à $1^{\prime}$ 'aérage. Le taux de défruitement de ces bandes fermes, compte tenu de ces galeries, a été fixé au maximum à $16 \%$. Les essais d'îlots réduits ont démarré sur ces bases au cours de 1 'année 77 et se poursuivent actuellement.

B) La rés istance mécanique du minerai concerné et les raisons d'être dé cés_éssais.

Différentes mesures de résistance en compression monoaxiale ont été entreprises sur le minerai de couche noire de Joudreville par le centre de Mécanique des Roches de 1 'Ecole des Mines de Paris (échantillons d'élancement 2).

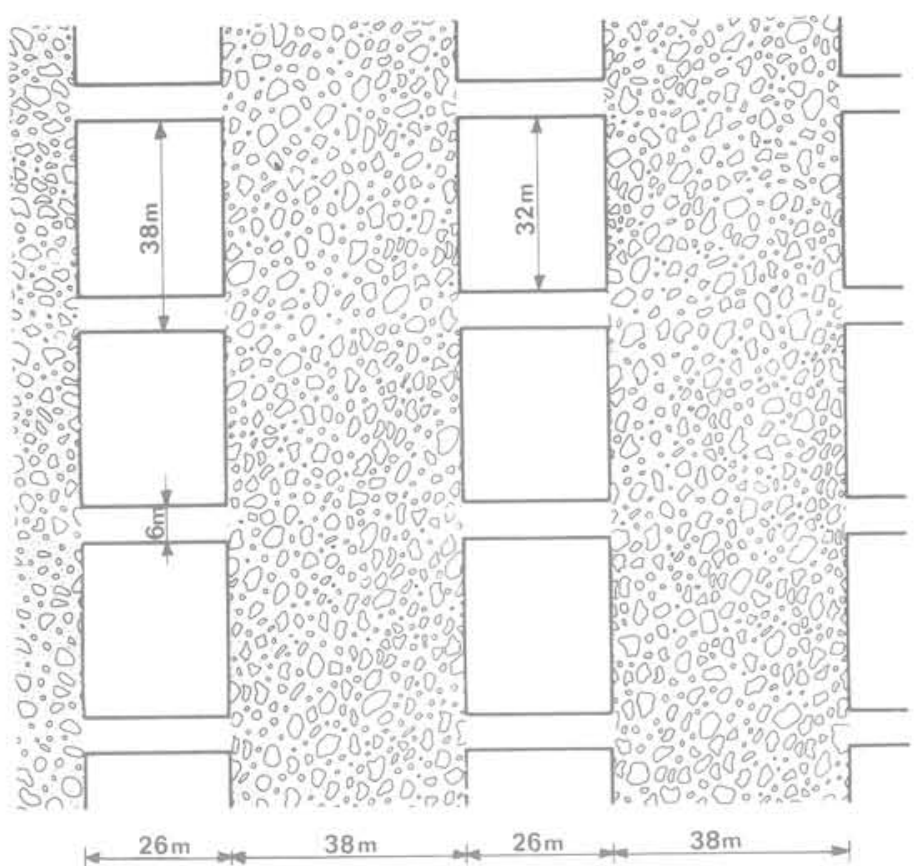

Taux de traf̧agóe bande ferme $=16 \%$

Taux de récupération sllobal $\cong 60 \%$

Taux d'ilotage $=\frac{38}{38+26} \cong 60 \%$

Fig.6 Schéma d'exploitation par flots réduits à Joudreville

\begin{tabular}{|l|c|c|c|}
\hline Lots & $\begin{array}{c}\text { résistance } \\
\text { instantanée } \\
\text { moyenne } \\
\mathrm{R}_{\mathrm{c} \text { en } 10^{6} \mathrm{~Pa}}\end{array}$ & $\begin{array}{c}\text { Ecart-type } \\
10^{6} \mathrm{~Pa}\end{array}$ & $\begin{array}{c}\text { Limite } \\
\text { élastique } \\
\mathrm{R}_{\mathrm{E}} \text { en } 10^{6} \\
\mathrm{~Pa}\end{array}$ \\
\hline $\mathrm{n}^{\circ} 1$ & 10,6 & 1,2 & 4,8 \\
$\mathrm{n}^{\circ} 2$ & 11,0 & 1,9 & 6,2 \\
$\mathrm{n}^{\circ} 3$ & 14,8 & 6,6 & 6,6 \\
$\mathrm{n}^{\circ} 4$ & 12,4 & 5,7 & 5,6 \\
Moyenne & 12,4 & - & 5,8 \\
générale & 12,4 & & \\
\hline
\end{tabular}

Ces valeurs dénotent un minerai particulièrement tendre. La moyenne des $R_{c}$ relevés dans le minerai de fer se situe aux environs de $22.10^{6} \mathrm{Pasca}$. La contrainte nature11e à $220 \mathrm{~m}$ de profondeur est de $\sigma_{z}=5,5,10^{6} \mathrm{~Pa}$ presque égale seulement à la lỉmite élastique $R_{F}$ de 1 a couche. Pour qu'une roche soit dépilable normalement avec foudroyage, l'expérience a montré que les inéquations suivantes devaient être satisfaites en règle générale :

$$
\begin{array}{r}
\mathrm{R}_{\mathrm{c}} \text { couche } \geqslant 3 \sigma_{\mathrm{z}} \\
3 \sigma_{\mathrm{z}} \leqslant \mathrm{R}_{\mathrm{c}} \text { toit } \leqslant 8 \sigma_{\mathrm{z}}
\end{array}
$$

En d'autres termes, si la couche et le toit sont trop tendres, les effets des pres- 
sions de terrain nées du dépilage sont incompatibles avec un soutènement classique par boulonnage. II faut faire appel en outre à la réduction des sections des galeries parfois même au cadrage de celles-ci. Ces procédés réalisables ont eux aussi leur limite et souvent ne peuvent pas être tolérés pour des raisons de coût dans de nombreuses mines. Si le toit est trop raide, on ne peut pas obtenir facilement le foudroyage. Ces valeurs 1 imites ne sont que des indications approximatives et auraient besoin d'être mieux précisées. Mais ces valeurs suffisent à montrer que le dépilage classique est très difficile dans la couche noire de la mine de Joudreville située à $200-220 \mathrm{~m}$ de profondeur.

Des essais de dépilage ont été cependant entrepris à Joudreville pour tenter d'exploiter ce minerai riche (37\% de Fe). Ils se sont soldés par des échecs complets. Le taux de défruitement ne dépassait pas $60 \%$ et le rendement du personnel d'abattage ne dépassait pas 60 tonnes par homme-poste, ce qui est un rendement très bas pour 1 es mines de fer. Ces performances lamentables étaient dûes non seulement aux déformations subies par les roches (minerai et toit) trop tendres, mais aussi par les venues d'eau provenant du foudroyage qui transformaient les roches également tendres du mur en un bourbier inextricable.

$$
0
$$

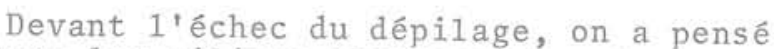
utiliser les piliers abandonnés ou leur variante : élargissage des galeries. Mais avec une résistance mécanique aussi basse, on ne pouvait espérer un taux de récupération convenable. En effet, un essai de découpage par piliers abandonnés au taux de $45 \%$ s'est terminé par I'écrasement du quartier.

Devant tous ces échecs et bien qu'il ne s'agissait pas d'un stot de protection, nous avons préconisé l'emploi de la méthode des îlots modifiée en îlots réduits pour supprimer les venues d'eau et pour diminuer le taux de contraintes s'exerçant sur les travaux d'exploitation entrepris à $1^{\prime}$ intérieur des illots (dans les bandes exploitées).

$$
\circ{ }^{\circ} \circ
$$

En accord avec 1a profession minière, avec 1 e Service des Mines et avec 1'aide de 1a DGRST, de Samifer et du centre de Mécanique des Roches de Fontainebleau, tout un programme d'essais, de mesures et de calculs a été entrepris pour essayer de sortir des règles empiriques précedemment énoncées. Nous reviendrons sur ce point. Auparavant, nous allons décrire beaucoup plus rapidement les essais entrepris simultanément dans d'autre mines à partir de I'année 1979.

$$
\circ \circ
$$

Disons pour terminer que les essais entrepris depuis 4 ans à Joudreville :

- ont été totalement couronnés de succès, - I'eau a disparu des travaux du fond, ce qui montre que les marnes micacées n'ont pas été atteintes par la "cloche" de foudroyage s'établissant sur chaque bande de largeur $\mathrm{L}=38 \mathrm{~m}$. Le foudroyage du toit suit bien le torpillage des piliers résidue $1 \mathrm{~s}$,

- les pressions de terrain ont notablement diminué. Les travaux d'exploitation sont aisés,

- les taux de défruitement s'établissent tout compris aux environs de $63 \%$,

- le rendement des mineurs est passé de 60 a plus de 130 tonnes par homme-poste,

- aucun affaissement de surface n'a été décelé à $1^{\prime}$ aplomb de la zone exploitée qui atteint désormais près de 30 hectares.

\section{II -2) Mine de Bazai11es}

Des raisonnements identiques à ceux qui ont présidé aux déterminations de Joudreville ont été utilisés à la mine de Bazailles.

I1 s'agit également d'une couche siliceuse relativement tendre de $3,5 \mathrm{~m}$ de puissance située à $170 \mathrm{~m}$ de profondeur.

Les essais de dépilage entrepris dans cette mine se sont soldés par des échecs dûs uniquement à l'eau qui transformait comme à Joudreville toutes les galeries et les voies d'accès en un bourbier traumatisant pour $1 \mathrm{e}$ personnel et bien érodant pour les engins miniers.

La méthode d'exp1oitation par piliers abandonnés a été utilisée pendant plusieurs années avec un taux de défruitement te1 que la stabilité pouvait être garantie pendant les travaux d'exploitation mais qui n'excluait pas $1^{\prime}$ effondrement des piliers dans un avenir plus ou moins lointain. Certains quartiers de piliers abandonnés se sont en effet progressivement effondrés entre un ou deux ans après 1'exploitation. D'autres tiennent encore actuellement après plusieurs années d'existence. Cette indétermination sur la date de 1 'effondrement consécutif à 1a fatigue des piliers n'est plus admise par le Service des Mines qui préconise des méthodes garantissant soit 1 'effondrement immédiat (cas du dépilage), soit la stabilité définitive.

La mine de Bazailles a donc été conduite à adopter 1a méthode des îlots réduits essentiellement pour se protéger des venues d'eau qui rendent inadmissibles les conditions d'exploitation.

$$
\begin{aligned}
& \text { On a adopté (voir fig. } \left.\mathrm{n}^{\circ} \mathrm{7}\right): \\
& \mathrm{L}=1 \text { argeur des bandes exploitées } \\
& \quad \text { formant les îlots }= \\
& \lambda=1 \text { argeur des bandes fermes }=
\end{aligned}
$$




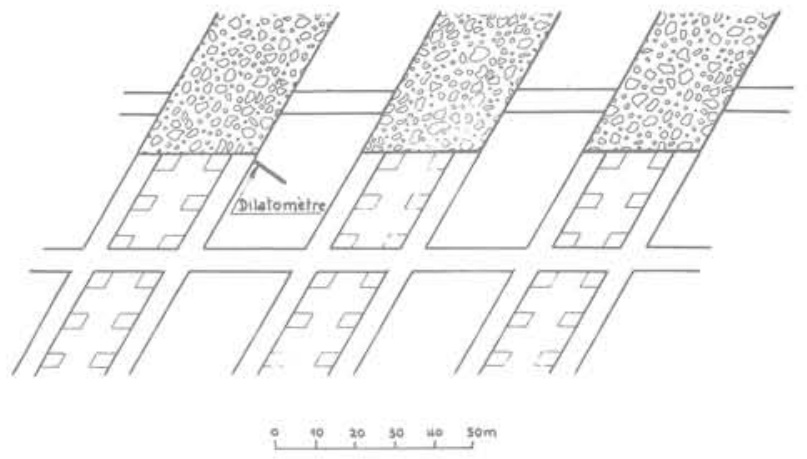

Fig.7 Schéma d'exploitation par 1lots réduits à Bazailles

1e taux d'ilotage :

$$
\tau=\frac{L}{L+\lambda}=55 \%
$$

Compte tenu des traçages effectués dans les bandes fermes et du taux de défruitement élevé des îlots, on arrive à un taux de défruitement global de $61 \%$.

Cette méthode conduit à Bazailles à des résultats d'exploitation excellents.

Les rendements abattage (concernant tout 1e personnel du quartier d'exploitation : foreur, mineur boulonneur, chargeur, camionneur, régie quartier) s'établissent aux environs de 200 tonnes par homme et par poste.

Aucun affaissement de surface n'a été relevé à 1'aplomb du quartier exploité par îlots réduits. Nous signalons cependant, à ce propos, que ce quartier ne fonctionne que depuis 15 mois. Il n'a pas encore subi une épreuve du temps suffisante pour être totalement affirmatif en ce qui concerne la stabilité définitive.

Enfin, le foudroyage des îlots se fait immédiatement après torpillage des piliers résidue1s Il est bien "complet". I1 y a donc formation d'une "cloche" de foudroyage. Cel1e-ci n'atteint pas les marnes micacées puisque $I^{\prime}$ on ne constate aucune venue d'eau au fond.

\section{II-1-3) La mine de Sêrouville}

Nous reviendrons plus en détail sur le cas de cette mine qui a effectué et qui effectue encore deux essais conduits simultanément :

- un essai d'îlot réduit,

- un essai de dépilage par taille avec soutènement marchant pour I'exploitation à l'intérieur des illots.

Dans ce paragraphe, nous ne donnerons que les résultats concernant les dimensions des îlots réduits qui ont été essayés dans cette mine.

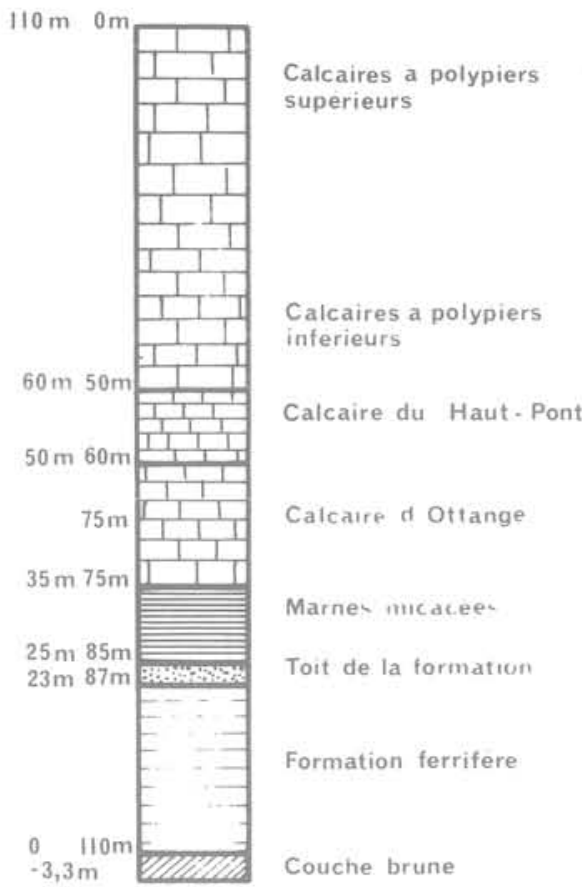

Fig. 8 Coupe du sondage de Serrouville

Celle-ci exploite une couche siliceuse de $3,5 \mathrm{~m}$ de puissance sous un faible recouvrement qui varie de 100 à 110 mètres.

Par contre, 1e toit immédiat de la couche exploitée est formé d'une marne compacte et épaisse qui nécessite pour son foudroyage une largeur d'exploitation $\mathrm{L}$ assez importante.

Les caractéristiques mécaniques de la couche exploitée sont les suivantes :

résistance en compression monoaxiale :

$$
\mathrm{R}_{\mathrm{C}}=18,010^{6} \mathrm{~Pa}
$$

limite élastique : $\mathrm{R}_{\mathrm{E}}=8,910^{6} \mathrm{~Pa}$

Les caractéristiques mécaniques du banc de marne formant le toit immédiat sont :

$$
\begin{aligned}
& \mathrm{R}_{\mathrm{C}}=22,810^{6} \mathrm{~Pa} \\
& \mathrm{R}_{\mathrm{E}}=10,610^{6} \mathrm{~Pa}
\end{aligned}
$$

- Etant donnée la faiblesse de I'épaisseur du recouvrement, $(100 \mathrm{~m})$ on était obligé d'adopter une largeur d'îlot $\mathrm{L}$ inférieure à $0,4 \mathrm{H}$ soit $40 \mathrm{~m}$ qui correspond à $1 \mathrm{a} 1 \mathrm{ar}-$ geur maximale d'un 1 lot classique, c'està-dire "non réduit".

- Etant donnée la proximité des marnes micacées dont le mur se situe à $28 \mathrm{~m}$ du mur de la couche exploitée (fig. $\mathrm{n}^{\circ} 8$ ) il ne fallait pas dépasser 33 à $35 \mathrm{~m}$ pour L si on voulait ne pas percer le banc de marnes micacées de 10 mètres d'épaisseur.

- Etant donnée 1a présence au toit immédiat d'un banc de marne compacte et épais (4 à $8 \mathrm{~m})$ et 1 a nécessité absolue d'obtenir un foudroyage pour les raisons que nous évo- 
querons plus loin, nous devions nous cantonner dans les largeurs maximales, c'està-dire 35 mètres.

Après plusieurs essais, nous avons admis que la meilleure largeur compatible avec les données du problème et avec une conduite aisée des travaux est celle de $35 \mathrm{~m}$ en orientant le fil de mine à $45^{\circ}$ par rapport au grand axe des îlots.

D'où: $\quad \begin{aligned} L & =35 \text { mètres } \\ \lambda & =22 \text { mètres }\end{aligned}$

Rappelons que nous reviendrons sur ces essais en parlant d'îlots réduits avec minitaille.

Disons dès maintenant que $1 \mathrm{a}$ méthode des ilots réduits n'a pas été mise en défaut lors de ces différents essais.

Même avec 1a 1argeur $\mathrm{L}=40 \mathrm{~m}$, les travaux d'exploitation n'ont subi aucune pression notable, aucune venue d'eau n'a été enregistrée jusqu'à maintenant. Par contre, des affaissements de surface très minimes ont été enregistrés à $1^{\prime}$ aplomb des îlots réduits. Ils semblent devoir se calmer avec le temps (voir fig. $\mathrm{n}^{\circ} 9$ ).

Par contre, pour les largeurs de $35 \mathrm{~m}$, aucun affaissement de surface n'a été enregistré.

\section{II-2) Détermination des 1argeurs L des îlots réduits}

II-2-1) Enoncé du problème à résoudre

Le problème posé est soumis à plusieurs contraintes :

1) il faut dans de nombreux cas déterminer la hauteur de $1 \mathrm{a}$ cloche de manière à ne pas atteindre $1 \mathrm{e}$ banc de recouvrement qui forme un écran imperméable vis-à-vis de tous les bancs aquifères susjacents.

2) il faut que Ia largeur $L$ soit cependant suffisante pour entraîner de façon impérative le foudroyage. Nous verrons que $1 \mathrm{a}$ stabilité des îlots dépend essentiellement de ce foudroyage qui vient "confiner" les zones périphériques des bandes fermes de largeur $\lambda$. Ces zones fortement sollicitées peuvent dans de nombreux cas sortir du domaine élastique et même se rompre. Ces zones, même rompues, conserveront un rôle porteur très important et constitueront un agent énergique de frettage du coeur du pilier à la condition qu'elles soient bloquées par un foudroyage aussi complet que possible (confinement)

3) I1 faut que 1a largeur L soit aussi réduite que possible de manière à minimiser la hauteur de la cloche, donc la hauteur (ou 1'épaisseur) des terrains qui viendront peser sur les travaux d'exploitation effec-

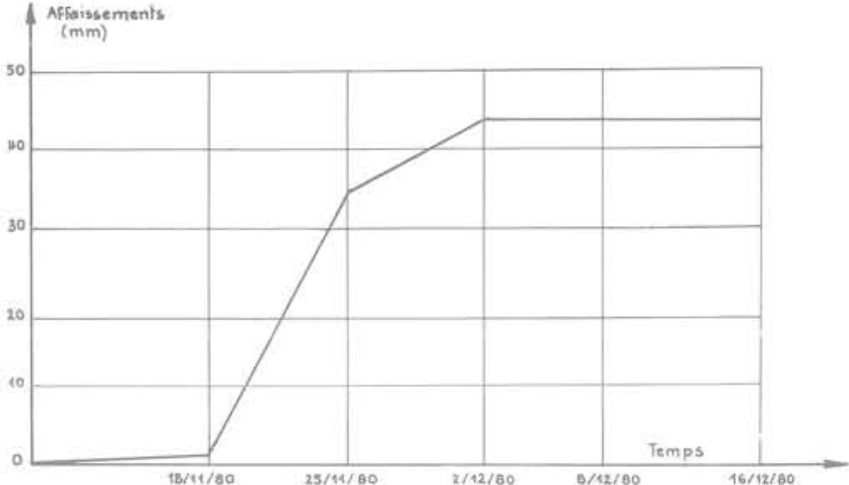

Fig.9 Affaissements de surface à 1 'aplomb de l'Ilot réduit de Serrouville

tués au sein des îlots. Cette dernière contrainte ne devient impérative que dans le cas où le minerai à exploiter (ou son toit) est tendre au point de ne pouvoir subir les sol1icitations nées d'une exploitation classique.

On voit donc que ces trois contraintes contradictoires peuvent dans certains cas définir des espaces disjoints et ne pas donner de solutions. En pratique, les problèmes d'exploitation ne sont pas si tranchés et on peut la plupart du temps donner des priorités aux différentes contraintes définies cidessus.

La solution de ce problème passe par 1'analyse du comportement mécanique des bancs immédiatement susjacents à $1 \mathrm{a}$ couche exploitée (bancs du toit immédiat).

I1 s'agit en fait de vérifier, pour une largeur L donnée, si les bancs du toit immédiats sont susceptibles de se rompre quand 1e minerai sous-jacent sera exploité. On détermine alors la hauteur atteinte par ces différents bancs rompus. Cette hauteur est-elle suffisante compte tenu du foisonnement pour remblayer la quasi-totalité de la cloche formée. D'autre part, cette hauteur est-elle délimitée par un banc (souvent dur ou épais) situé en dessous du banc imperméable que $1^{\prime}$ on veut préserver ?

\section{I-2-2) Etude de 1a stabilité des bancs du toit immédiat. Détermination de 1 a haut eur des cloches}

\section{A) Principe des calculs et résultats}

Pour étudier la stabilité des bancs du toit immédiat, nous supposerons qu'ils ne s'effondrent pas à $1^{\prime}$ aplomb de la zone exploitée dans l'îlot rédu亡t. Nous abtenons alors une galerie (voir fig. $\mathrm{n}^{\circ}$ 10) de largeur $\mathrm{L}$ surmontée des bancs $\mathrm{B}_{1}, \mathrm{~B}_{2}, \mathrm{~B}_{3} \ldots \mathrm{B}_{\mathrm{n}}$. Connaissant les caractéristiques géométri-n ques et mécaniques de ces différents bancs, en particulier les épaisseurs,1es différents modules d'élasticité, coefficients de Poisson, résistances en compression instantanée, 


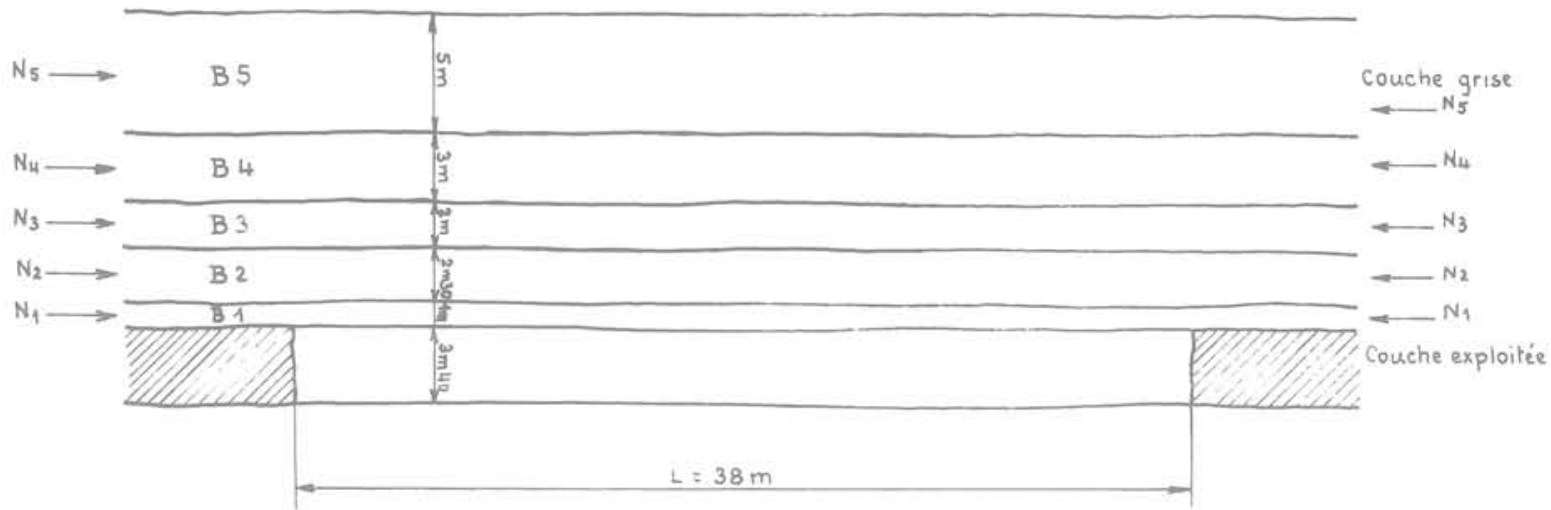

Fig.10 Schématisation des bancs situés à l'aplomb d'un îlot à Joudreville

limites élastiques, etc..., nous pouvons tenter d'évaluer leur stabilité. Le principe et le détail des calculs ont été exposés dans notre article intitulé "MODE D'ACTION ET REGLES DU BOULONNAGE" - Revue de 1'Industrie Minérale - Série Mine - $\mathrm{N}^{\circ}$ Spécial Boulonnage - tome II - 1978.

Appliquons ces calculs aux îlots réduits de Joudreville. Les caractéristiques géométriques et mécaniques des différents bancs $\mathrm{B}_{1}, \mathrm{~B}_{2}, \mathrm{~B}_{3} \ldots$ sont les suivantes :

\begin{tabular}{|c|c|c|c|c|c|}
\hline Banes & $\begin{array}{c}\text { Epafiseur } \\
\text { on metres } \\
\text { m }\end{array}$ & $\begin{array}{l}\text { Ressistance en } \\
\text { courression } \\
\text { Res }\end{array}$ & $\begin{array}{c}\text { Lititice 61astique } \\
\text { on compression } \\
n_{E}\end{array}$ & $\begin{array}{c}\text { Module } \\
\text { d.elasticite } \\
\text { E }\end{array}$ & $\begin{array}{c}\text { Coefficient } \\
\text { de Poisson } \\
y\end{array}$ \\
\hline$\stackrel{\mathrm{B}_{1}}{\text { (marnes) }}$ & $1,0 \pi$ & $10.10^{6} \mathrm{~Pa}$ & $5,5.10^{6} \mathrm{~Pa}$ & $5000.10^{6} \mathrm{~Pa}$ & 0,25 \\
\hline $\begin{array}{l}{ }^{\mathrm{F}} \\
\text { (minerai } \\
\text { giliceux } \\
\text { ot pauvre) }\end{array}$ & $2,3 \mathrm{~m}$ & $11.10^{6} \mathrm{~Pa}$ & $5,2.10^{6} \mathrm{PA}$ & $8000.10^{6} \mathrm{~Pa}$ & 0,2 \\
\hline $\begin{array}{c}\mathrm{D}_{3} \\
\text { (marnes) }\end{array}$ & $2,0 \mathrm{~m}$ & $10.10^{6} \mathrm{~Pa}$ & $5,5.10^{6} \mathrm{~Pa}$ & $5000.10^{6} \mathrm{Fa}$ & $n, 25$ \\
\hline $\begin{array}{c}n_{4} \\
\text { (marnes) }\end{array}$ & $3,0 \mathrm{~m}$ & $10.10^{6} \mathrm{~Pa}$ & $5,5.10^{6} \mathrm{~Pa}$ & $5000.10^{6} \mathrm{~Pa}$ & 0,25 \\
\hline $\begin{array}{c}B_{5} \\
\text { (marnes) }\end{array}$ & $5,0 \mathrm{~m}$ & $25.10^{6} \mathrm{~Pa}$ & $12,0,0^{6} \mathrm{~Pa}$ & $10000,10^{6} \mathrm{~Pa}$ & 0,2 \\
\hline
\end{tabular}

Rappelons très sommairement le principe des calculs qui prend en compte séparément chacun des bancs qui sont sollicités en flexion complexe sous $1^{\prime}$ action de leur propre poids et sous l'action des poussées horizontales $\mathrm{N}_{1}, \mathrm{~N}_{2}, \mathrm{~N}_{3}$ engendrées par 1a contrainte naturelle horizontale qui règne dans le massif, notablement renforcée par 1'existence même de la galerie. Il s'agit donc de dalles encastrées au droit des parements de la galerie de largeur L et travaillant en flexion complexe. Cette façon d'opérer est justifiée par le fait couramment observé à 1 'endoscope : 1es bancs $\mathrm{B}_{1}$, $\mathrm{B}_{2}$ et $\mathrm{B}_{3}$ fléchissent indépendamment les uns des autres au point de présenter des vides, dits vides de décollement, entre chaque plan de stratification.

Par ailleurs, tous ces bancs étant fissurés naturellement (fil de mine en particulier) il ne saurait être question d'obtenir des tractions. Les moments ne peuvent engendrer que des compressions. Les efforts tranchants peuvent s'exercer en tendant à faire glisser les éléments découpés par les fissures naturelles. En appliquant ces principes de calcul, on trouve que 1e banc B5 (couche grise) ayant 5 mètres d'épaisseur devrait constituer le sommet de la cloche.

Tous les bancs inférieurs subissent des contraintes de compression nettement supérieures à $R_{c}$. Si tel est bien le cas, le sommet de $1_{a}$ cloche devrait se situer à $11,70 \mathrm{~m}$ du mur de la couche noire exploitée et le foudroyage devrait pratiquement remplir toute la cloche. En effet, $8,30 \mathrm{~m}$ de bancs $\left(B_{1}+B_{2}+B_{3}+B_{4}\right)$ vont rompre donc se foudroyer. La densitê en place de ces bancs étant voisine de 2,50 , ils vont se présenter après foudroyage avec une densité foisonnée de 1,8 légèrement supérieure à $1 \mathrm{a}$ densité foisonnée courante des roches abattues par le tir qui est de 1,6. En effet, ce foudroyage sur plus de $10 \mathrm{~m}$ d'épaisseur subit au moins à sa base un tassement sous son propre poids. L'ensemble de ces bancs rompus vont alors occuper une hauteur dans la cloche approximativement égale à :

$$
8,3 \times \frac{2,5}{1,8}=11,5 \text { mètres }
$$

On devrait donc s'attendre à un remplissage à peu près complet de la cloche formée.

On obtient de cette manière, à $1 \mathrm{a}$ mine de Joudreville, où $L$ et $\lambda$ ont été fixés respectivement à 38 et $26 \mathrm{~m}$ :

- des gros piliers ou bandes fermes de largeur $\lambda=26 \mathrm{~m}$ ayant $12 \mathrm{~m}$ de hauteur environ soit un élancement très favorable de :

$$
\frac{12}{26}=0,46
$$

- des "cloches" dont le sommet se trouve à 12 mètres environ du mur de 1 a couche exploitée. Ce sommet est très éloignée des marnes micacées qui sont situées à $37,5 \mathrm{~m}$ de hauteur.

- un autoremblayage presque complet de ces piliers d'élancement 0,46 grâce au foudroyage des cloches situées de part et d'autre de chacun d'eux. 
Des calculs identiques ont été effectués dans chacune des mines pratiquant les illots réduits, ainsi qu'à la mine de Droitaumont où les illots classiques ont été constitués.

Dans cette dernière mine nous avons trouvé par le calcul une hauteur de cloche de $50 \mathrm{~m}$ environ, ou plutôt nous avons vérifié que le banc situé à $50 \mathrm{~m}$ et qui constituait le sommet de $1 \mathrm{a}$ cloche observée par sondage devait effectivement résister étant donné son épaisseur et ses caractéristiques mécaniques.

Les ilots de Drautaumont avaient une largeur de $\mathrm{L}=60 \mathrm{~m}$ pour une hauteur de recouvrement de 170 mètres.

$$
\circ \circ
$$

Nous remarquons qu'il faut connâtre les caractéristiques mécaniques et géométriques de chaque banc situé à l'aplomb immédiat de la couche où les îlots vont être constitués.

Pour cela, nous avons utilisé l'appa- $^{\prime}$ reil mis au point par le centre de Mécanique des Roches de l'Ecole des Mines de Paris, sur une idée de Monsieur FINE. I1 s'agit du SAIGNOFOR qui permet de relever en continu la résistance en compression des roches traversées par un sondage. Ce sondage avait été foré à notre demande pour vérifier la hauteur de 1a cloche (voir § suivant).

Nous donnons (fig. $\mathrm{n}^{\circ}$ 11) le diagramme des résistances en compression tout $1 \mathrm{e}$ long du sondage qui a été foré à $1^{\prime}$ aplomb d'un des îlots de la mine de Droitaumont. Le sondage avait percé dans le cloche de foudroyage à la cote 120 mètres et le relevé des résistances en compression a été effectué de 1 a cote 120 à la cote 60 .

B) Confrontation des résultats des calculs_de hauteur de cloche avec les observations et les mesures

Pour vérifier la validité de nos raisonnements ainsi que la validité de nos calculs relatifs au sommet des cloches, nous avons effectué deux sondages à $I^{\prime}$ aplomb d'îlots constitués dans les mines de fer de Lorraine.

1) 1'un des sondages, celui effectué à 1a mine de Droitaumont, devait déboucher à l'aplomb d'un îlot classique dont 1a largeur L avait été déterminee par la formule empirique :

$$
\mathrm{L} \leqslant 0,4 \mathrm{H} \text { avec } \mathrm{H}=170 \mathrm{~m}
$$

Ce11e-ci conduisait à une largeur d'îlots de $68 \mathrm{~m}$ qui fut ramené à $60 \mathrm{~m}$ pour diverses raisons. Ces îlots avaient été réalisés il y a 20 ans environ.

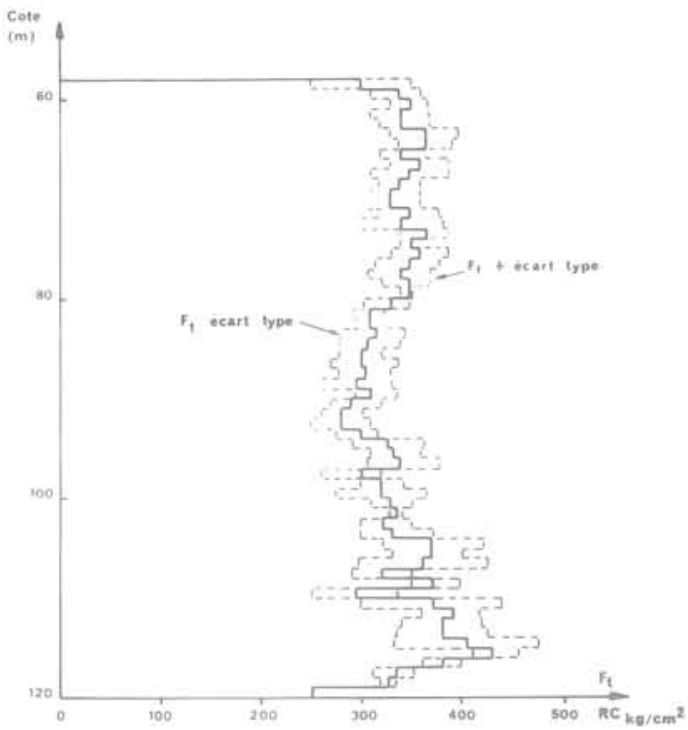

Fig.11

2) Le deuxième sondage a été foré à $1 \mathrm{a}$ mine de Joudreville à l'aplomb d'un îlot réduit de largeur $\mathrm{L}=38 \mathrm{~m}$, situé à cet endroit précis à une profondeur $\mathrm{H}$ de $220,5 \mathrm{~m}$ (se reporter à $1 \mathrm{a}$ coupe de $1 \mathrm{a}$ figure 5).

Ces sondages de $30 \mathrm{~cm}$ de diamètre ont été auscultés sur toute leur profondeur grâce à des caméras électroniques étanches, enregistrant sur bandes magnétiques les images de 1a paroi des sondages. Ces images permettent de relever même dans les trous immergés, de mettre en évidence des fissures de moins de $1 \mathrm{~mm}$ d'épaisseur.

Tout cet appareillage a été mis au point par SAMIFER qui exécute une auscultation complète d'un sondage de $200 \mathrm{~m}$ en quelques heures. Les sommets de cloches ont été très bien observés avec un vide entre ce sommet et le foudroyage de $0,5 \mathrm{~m}$ à $1 \mathrm{~m}$ environ.

Les résultats obtenus sont résumés dans

\begin{tabular}{|c|c|c|}
\hline & Droitaumont & Joudreville \\
\hline $\mathrm{H}$ & $170 \mathrm{~m}$ & $220,5 \mathrm{~m}$ \\
\hline L & $60 \mathrm{~m}$ & $38,0 \mathrm{~m}$ \\
\hline \multicolumn{3}{|c|}{$\begin{aligned} h= & \text { hauteur des clo- } \\
& \text { ches depuis le } 1 \\
& \text { mur de la couche } \\
& \text { exploitée }\end{aligned}$} \\
\hline $\begin{aligned} h^{\prime}= & \text { hauteur des de } \\
& \text { niers vides de } \\
& \text { décollement }\end{aligned}$ & 47 & $20,0 \mathrm{~m}$ \\
\hline $\mathrm{h} / \mathrm{L}$ & $\frac{47}{60}=0,78$ & $\frac{12}{38}=0,32$ \\
\hline$h^{\prime} / L$ & $\frac{47}{60}=0,78$ & $\frac{20}{38}=0,53$ \\
\hline
\end{tabular}
le tableau suivant : 
Les calculs proposés donnent des résultats en accord avec les observations.

Il semblerait toutefois que les bancs stables du sommet de la cloche qui se "décollent" des bancs susjacents finissent, avec le temps, par se rompre, sans doute par effet de vieillissement de la roche intéressée. En effet, $h$ et $h$ ' sont confondus à l'aplomb des cloches ayant 20 ans d'âge à Droitaumont, et on décèle, à Joudreville, des plans de décollement au sommet des c1oches n'ayant qu'une année d'existence.

I1 est facile de tenir compte de cette nouvelle contrainte dans les calculs. En pratique, cela revient à dire que le sommet des cloches s'établit sous un banc relativement dur, mais surtout épais, éventuellement surmonté de bancs plus minces qui suivront la déformation (sans décollement) du banc épais tout en contribuant à l'augmentation de son moment d'inertie et de sa résistance en compression.

II-3) Détermination des 1argeurs $\lambda$ des bandes fermes-séparant les îlots

Plusieurs voies de recherche ont été tentées successivement au centre de Mécanique des Roches de $1^{\prime} E c o l e$ des Mines de Paris.

\section{II-3-1) Tentative d'évaluation des largeurs $\lambda$ grâce à 1 éffet de forme des ban- des fermes}

Nous avons vu que $1^{\prime}$ élancement des "piliers" formant les bandes fermes à Joudreville était égal à 0,46 . Nous avons donc supposé'tout d'abord que 1 a bonne stabilité constatée avec les îlots était dûe à la forme très favorable des bandes fermes, ce qui n'était pas toujours le cas avec les piliers $\mathrm{ab}$ andonnés.

Une étude menée par Monsieur Boucly au centre de Mécanique des Roches de $1^{\prime}$ Ecole des Mines de Paris a quantifié $1^{\text {'effet de }}$ Forme pour le minerai de fer de Lorraine.

L'essentiel des résultats obtenus concerne des minerais de fer prélevés à la mine de Saizerais et a la mine de Joudreville.

Appelons h 1a hauteur de I'éprouvette $\ell$ la largeur de 1'éprouvette

L'élancement est donné par $\mathrm{h} / \mathrm{l}$

L'anti élancement est donné par $\ell / h$

On a en outre défini un facteur de forme $F$ ou valeur de la grandeur mesurée (par exemple, la résistance instantanée en compression) pour un élancement donné, ramené à la valeur obtenue pour 1'élancement unité ou pour l'élancement 2 servant de référence.

Le facteur de Forme en résistance instantanée en compression est :

$$
F=\frac{R c i}{R c_{1}}
$$

Rci : résistance instantanée en compression obtenue pour l'anti élancement i

$\mathrm{Rc}_{1}$ : résistance instantanée en compression obtenue pour $1^{\prime}$ anti élancement 1 .

\begin{tabular}{|c|c|c|c|c|}
\hline \multicolumn{5}{|c|}{ Limite élastique longitudinale - RL } \\
\hline & \multicolumn{4}{|c|}{ t/e } \\
\hline $\begin{array}{l}\text { Elancegenat } \\
\text { Anti-étancesent }\end{array}$ & $0_{0}^{2}$ & 1 & 0,5 & 0,33 \\
\hline 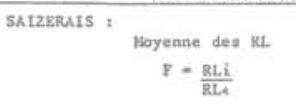 & $\begin{array}{l}17,0.10^{6} \mathrm{~Pa} \\
0.76\end{array}$ & $\begin{array}{l}22,5 \cdot 10^{6} \mathrm{~Pa} \\
1\end{array}$ & $\begin{array}{c}28,7,10^{6} \mathrm{~Pa} \\
1,27\end{array}$ & $\begin{array}{l}35,8.10^{6} \mathrm{~Pa} \\
1.59\end{array}$ \\
\hline 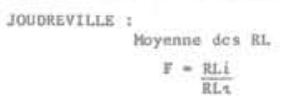 & $\begin{array}{l}14,5 \cdot 10^{6} \mathrm{pa} \\
0.79\end{array}$ & $18,4.10^{6} \mathrm{~Pa}$ & $\underset{1,25}{23,0.10^{6} \mathrm{pa}}$ & $\begin{array}{l}28,85.106 \mathrm{p} \\
1,57\end{array}$ \\
\hline
\end{tabular}

\begin{tabular}{|c|c|c|c|c|}
\hline \multicolumn{5}{|c|}{ Résistance à la rupture en compression - Rc } \\
\hline \multirow[b]{2}{*}{ 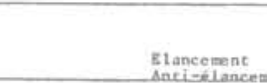 } & \multicolumn{4}{|c|}{$h / t$} \\
\hline & $\sum_{0,5}^{2}$ & $i$ & 0,5 & 0,33 \\
\hline SATZERATS : & & & & \\
\hline $\begin{array}{c}\text { Muyvume des Re } \\
p-\frac{\mathrm{Rec}}{\mathrm{Ret}}\end{array}$ & $\begin{array}{l}23.6,10^{6} \mathrm{ra} \\
0.75\end{array}$ & $31,1,10^{6} \mathrm{~Pa}$ & ${ }_{1,15}^{35,8.10^{6} \mathrm{~Pa}}$ & $\frac{55,0,10^{6} \mathrm{r}+1}{1,77}$ \\
\hline JOUDREVLLLE : & & & & \\
\hline $\begin{array}{c}\text { Moyenne des Rc } \\
F-\frac{R c i}{R c i}\end{array}$ & $\begin{array}{l}27,2,10^{6} \mathrm{~Pa} \\
0,97\end{array}$ & ${ }_{1}^{28,1}, 10^{6} \mathrm{pa}$ & ${ }_{1,81}^{31,8.10^{6} \mathrm{~Pa}}$ & $\begin{array}{l}39,2.10^{6} \mathrm{~Pa} \\
1.4\end{array}$ \\
\hline
\end{tabular}

Nous nous intéressons surtout aux $1 \mathrm{imi-}$ tes élastiques puisque les bandes fermes ont à subir des efforts permanents d'une durée illimitée.

En partant du facteur de forme relatif à la limite élastique, on trouve approximativement pour les deux types de minerai :

$$
\frac{R L_{i}}{R L_{1}} \cong 0,69+0,31 \frac{\ell}{h}
$$

Si nous prenons comme référence les résultats obtenus sur les éprouvettes d'élancement 2 (anti élancement 0,5 )

$$
\frac{\mathrm{RLi}}{\mathrm{RL} 0,50} \cong 0,8+0,4 \quad \frac{\ell}{\mathrm{h}}
$$

Ces relatinns sont certainement approximatives car reposant sur des essais trop peu nombreux. E1les n'ont été vérifiées que dans le domaine

$$
0,5 \leqslant \frac{l}{h} \leqslant 3
$$

L'application des relations précedentes au cas de Joudreville conduirait à un taux d'ilotage de $48 \%$. Si tel était le cas, il faudrait adopter une largeur $\lambda$ des bandes fermes égale à 41 mètres si nous adoptons une largeur d'ilot réduit de $\mathrm{L}=38$ mètres. 
Comme les essais in situ ont montré que la stabilité était acquise (sur une durée de 4 ans) pour un taux de défruitement de $63 \%$ et un $\lambda$ de $26 \mathrm{~m}$, nous en concluons que la stabilité constatée des îlots ne semble pas dûe à l'effet de forme des "piliers" constitués par les bandes fermes.

\section{II -3 -2) Ana1yse des contraintes s'exerçant dans les bandes fermés}

Des calculs entrepris par éléments finis en élasticité et en élastoplasticité au centre de Mécanique des Roches de 1'Ecole des Mines de Paris ont montré (fig. $n^{\circ} 12$ ) qu'avec les caractéristiques des minerais 1orrains, les zones périphériques des bandes fermes, sur plusieurs mètres d'épaisseur, étaient toujours rompues ou étaient sorties du domaine élastique quelles que soient les formes de cloches observées ou supposées. Les conclusions de ces calculs étaient les suivantes :

1) le schéma retenu dans le paragraphe précédent consistant à rechercher une forme adéquate des "piliers" constituant les bandes fermes pour que ceux-ci restent en tout point en élasticité n'était pas celui qui devait être pris en compte pour la détermination des largeurs $\lambda$. Certainement le taux de charge imposé aux "piliers" découpés en pratique au fond les obligeait à subir des ruptures dans certaines zones ou les obligeait à sortir du domaine élastique.

2) Sans soutènement et sans confinement des zones rompues ou plastifiées, on était certain d'obtenir 1'instabilité donc 1 a ruine des bandes fermes.

I1 devenait donc évident que ce confinement et ce soutènement étaient en fait réalisés par la présence du foudroyage obtenu à la suite de $1^{\prime}$ exploitation au sein des îlots. Celui-ci forme un auto-remblayage des "piliers" constitués par les bandes fermes. on était donc amené à envisager les calculs de stabilité de ces bandes fermes en tenant compte de ce fait.

II-3-3) Mesures de déformations effectuées. au sein des bandes fermes

I1 nous restait encore à prouver que les zones périphériques des bandes fermes rentraient bien soit en rupture soit en plasticité. Pour cela, SAMIFER mettait en place au sein des bandes fermes des mesureurs de dilatation ou d'expansion ancrés à diverses profondeurs au niveau de la couche exploitée.

Ces mesures avaient pour but de :

a) préciser l'étendue des zones périphériques qui se rompaient ou sortaient du domaine élastique en se dilatant.

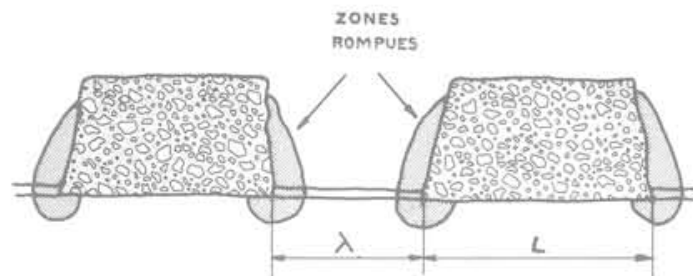

Fig.12 Zones rompues ou plastifiées en périphérie des bandes fermes

b) quantifier 1'évolution de la "dilatance" de cette zone rompue.

c) déceler 1'existence de zones centrales qui normalement frettées par les zones rompues en dilatance qui prenant appui sur le foudroyage devaient comprimer dans le sens horizontal le coeur des bandes fermes.

Les relevés devaient être effectués par télémesure à longue distance et sur une Iongue période. En effet, 1 'accès aux appareils était rendu impossible par le foudroyage. I1 fallait de plus placer les appareils de mesure et débuter les mesures avant le passage des fronts d'exploitation au sein des deux ilots contigüs puis attendre $1 \mathrm{a}$ fin de ces exploitations.

La figure 13 donne 1 a disposition des appareils et le relevé de ces mesures de déformation horizontales entreprises au sein de 1 'une des bandes fermes de 26 mètres de largeur à Joudreville et entreprises avant, pendant et après $1^{\prime}$ exploitation des îlots contigüs.

On voit qu'au niveau de la couche exploitée 1a zone rompue au sein de la bande ferme se dilate vers le foudroyage sur une épaisseur qui doit être de 1 'ordre de 3 mètres environ. La zone centrale quant à elle subit une déformation de tassement.

On notera que le montant des déformations de dilatation soit 40 millimètres obtenues à la fin de l'exploitation des ilots et intéressant une épaisseur de roche au plus égale à 3 mètres prouve que $1 \mathrm{a}$ roche intéressée est certainement sortie du domaine élastique.

Cette dilatance de 40 millimètres constitue si l'on peut dire l'agent provoquant le tassement du foudroyage et du coeur de 1 a bande ferme.

II -3-4) Relevé des caractéristiques mécaniques du minerai rompu en fonction des _contraintes déconfinement.

A la suite de toutes ces observations, de toutes ces mesures de déformation et d'affaissement de surface, nous pouvions affirmer :

- que les bandes fermes étaient stables puisqu'il n'y avait aucun affaissement de surface, 


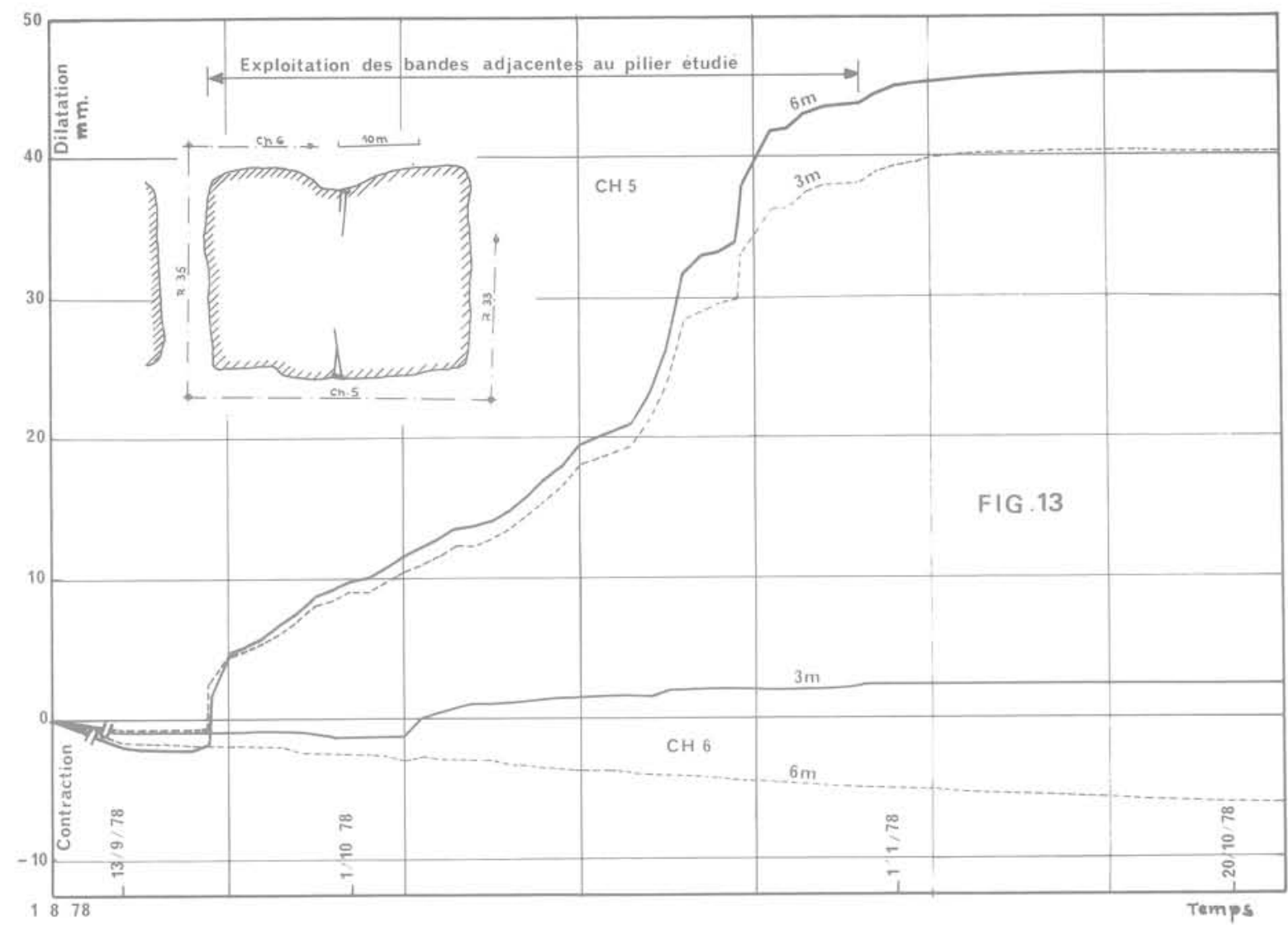

Fig.13 Mesures de dilatations à la mine de Joudreville

Revenons au problème du comportement du minerai de fer parvenu à la rupture et soumis à confinement.

Pour ce faire, le Centre de Mécanique des fermes était tel que leur zone périphérique était rompue sur plusieurs mètres,

- que leur zone centrale restait dans le domaine élastique et travaillait en triple étreinte car fretté par la présence des zones rompues en dilatance, elle-mêmes confinées par la présence du foudroyage.

Ce schéma de mise en charge et de comportement des bandes fermes étant connu, on pouvait prétendre au calcul de celles-ci à condition de connaitre :

-1e comportement du minerai et des bancs du toit immédiat lorsqu'ils sont sollicités en triple étreinte. Les essais triaxiaux classiques donnent ies renseignements correspondants.

- le comportement des mêmes roches une fois rompues en fonction de 1 'intensité de la pression de confinement. C'est $1^{\prime}$ objet du présent paragraphe.

- le comportement du foudroyage soumis à des sollicitations en triple étreinte de faible intensité. Cette partie de 1 a recherche n'est pas encore effectuée. Nous verrons dans le paragraphe suivant que nous pouvons nous contenter pour 1 'instant d'introduire dans les calculs de bande le comportement du foudroyage sous forme paramétrique. des Roches de l'Ecole des Mines de Paris a entrepris (Messieurs VOUILLE et SAGHAFI) une série d'essais de mise en charge d'éprouvettes de minerai de fer, d'élancement 2, soumises en outre à des pressions de confinement variant entre $0,2,10^{6}$ et $0,8,10^{6}$ Pascal.

Grâce à un montage réalisé sur place, on a augmenté la rigidité de la presse de manière à contrôler le développement de $1 \mathrm{a}$ fracturation.

Nous donnons figure 14 1'un des relevés des courbes effort-déformation longitudinale obtenues avec ce montage et montrant 1'existence après rupture d'un écrouissage négatif suivi d'une déformation plastique sans écrouissage. Le pallier correspondant à ces déformations plastiques sans écrouissage (partie horizontale de 1a courbe effort-déformation) s'établit pour un niveau de mise en charge d'autant plus élevé que la pression de confinement est plus forte.

Grâce à 1'analyse de ces courbes, on a pu obtenir les caractéristiques du minerai rompu en particulier :

- I'angle de frottement

- les seuils à partir desquels on entre en plasticité à écrouissage négatif puis en plasticité sans écrouissage. 


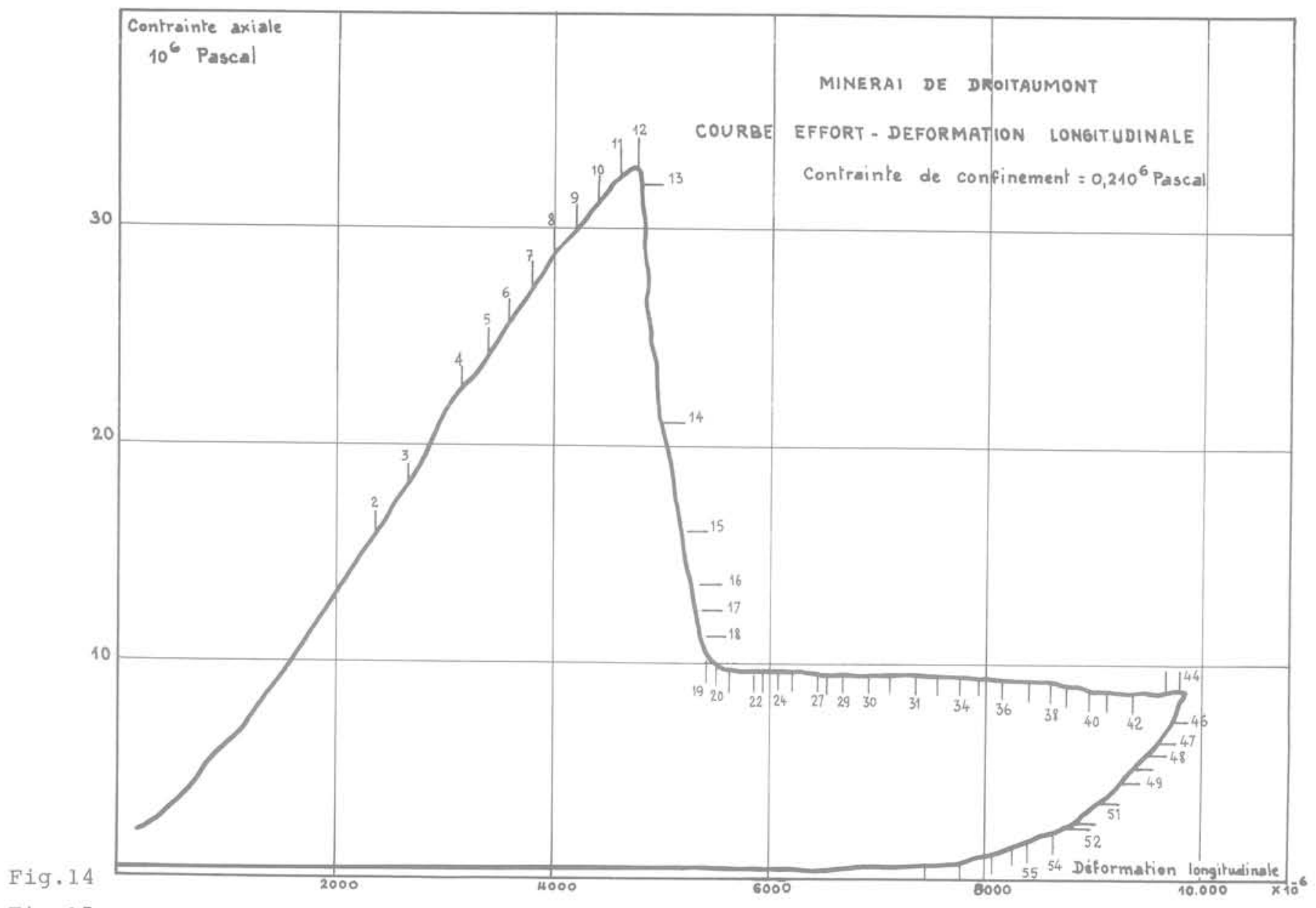

Fig. 15

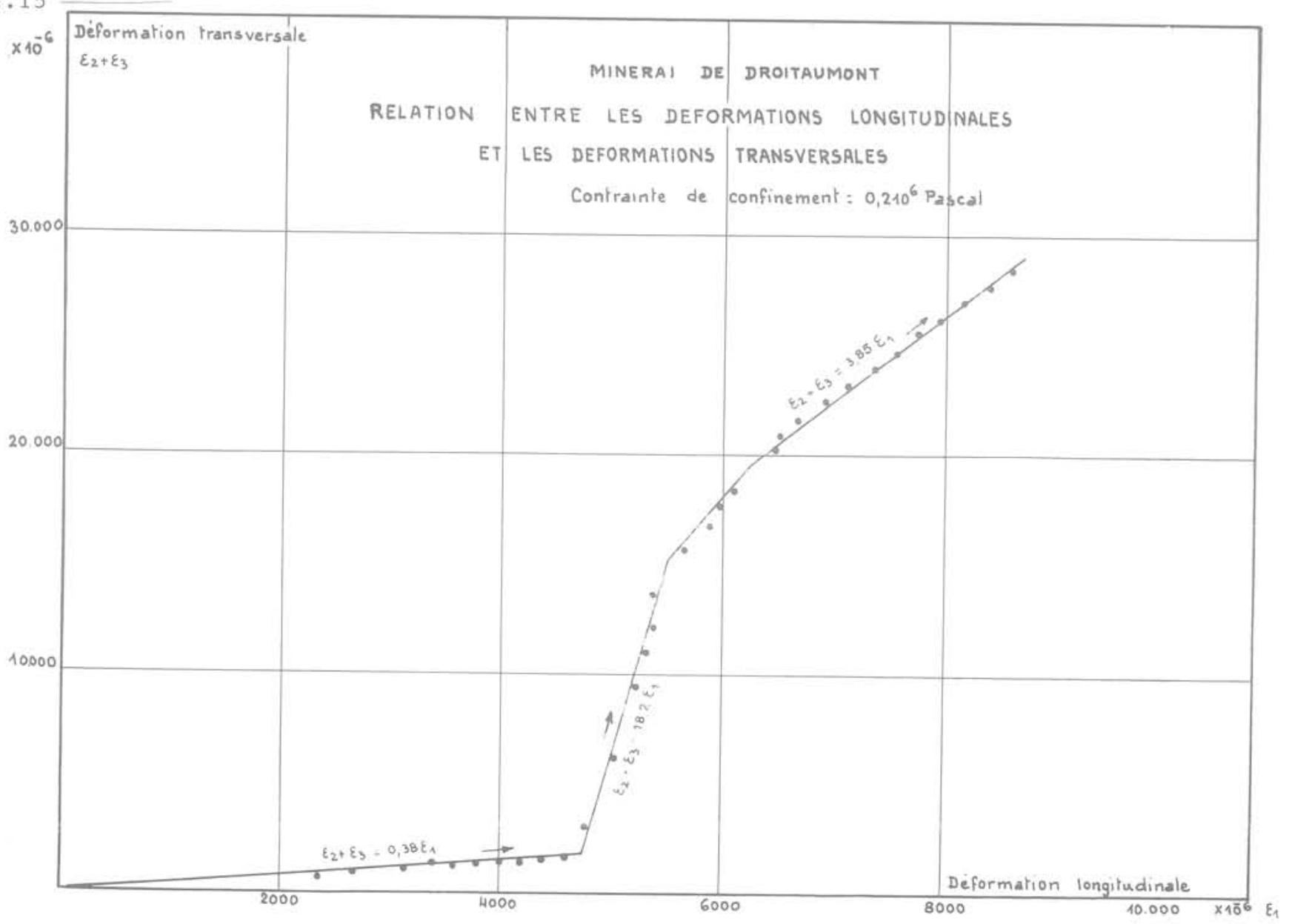


On a relevé en outre (fig. $n^{\circ} 15$ ) et en même temps les courbes effort-déformation transversale au cours des essais décrits cidessus nous permettant d'obtenir les valeurs des déformations horizontales qui sont à 1'origine de la dilatance, du confinement et du frettage du coeur des bandes fermes.

I I-3-5) Principe du calcul des bandes fermes. Mise au point du programme de calcul.

Le calcul réalisé par 1 a méthode des éléments finis utilise le programme VIPLEF mis au point au centre de Mécanique des Roches de 1 'Ecole des Mines de Paris par S.M. TIJANI.

Le comportement du minerai (et des roches constitutives des bancs du toit intéressés par la banile ferme), y compris la phase post-rupture, est représenté par un modèle élasto-p1astique à écrouissage négatif. On a adopté cette représentation car elle rend bien compte des essais que nous venons de décrire. De plus, elle a été utilisée avec succès pour simuler $1 \mathrm{a}$ mise en charge du revètement des tunnels profonds dans un cas où le calcul analytique était praticable (PANET Marc, 1976, Analyse de la stabilité d'un tunnel creusé dans un massif rocheux en tenant compte du comportement après la rupture.

Rock Mechanics, vol. 8, $\mathrm{n}^{\circ} 4$, pages 209-223)

En 1'absence de résultats expérimentaux très difficiles à obtenir, le comportement du foudroyage est simulé par un modèle élastoplastique dont les caractéristiques sont les caractéristiques résiduelles de la roche du toit. C'est en particulier un matériau à cohésion nulle.

L'algorithme de calcul est de type itératif. Supposant connus le chargement, les déformations plastiques en tous points (elles peuvent éventuellement être nulles), et en tous points également une quantité définissant l'écrouissage du matériau, on calcule par la méthode des éléments finis, en élasticité avec contrainte initiale, les contraintes s'exerçant en tous points.

Si ces contraintes ne violent pas $1 \mathrm{e}$ caractère de plasticité, on ne modifie ni les déformations plastiques, ni la variable d'écrouissage. Dans tous les points où il en est autrement, 1 a relation de $1^{\text {'élasto- }}$ plasticité permet de définir de nouvelles déformations plastiques et une nouvelle variable d'écrouissage. On reprend alors le calcul avec ces nouvelles variables jusqu'à ce que les variations provoquées par une itération nouvelle soient négligeables.

Ce nouveau programme de calcul est maintenant au point et nous I'appliquons actue 1lement au calcul des bandes fermes dans les trois mines ayant conduit les essais d'îlots réduits in situ.

Pour l'instant, ces calculs manquent de précision car nous ignorons encore les vraies caractéristiques du foudroyage. IIs nous donnent cependant les valeurs de la largeur cherchée en fonction de paramètres adoptés plus ou moins arbitrairement pour caractériser le foudroyage.

Nous comptons bien entreprendre prochainement les essais nécessaires in situ pour mesurer les propriétés mécaniques du foudroyage.

\section{II-3-6) Le contrôle de 1a stabilité des îlots}

$\mathrm{Si}$ nous voulons assurer absolument $1 \mathrm{a}$ stabilité de la surface, il faut constituer des illots conformes aux dimensions établies suivant les modalités précédentes jusqu'à plus ample informé.

Mais tout calcul doit être contrôlé par des observations et mesures en surface et au fond.

Le critère de stabilité parfaite revient à admettre que les zones rompues ou plastifiées en périphérie des bandes fermes ne viennent pas se rejoindre au coeur de celles-ci. En d'autres termes, il faut qu'il existe toujours dans 1a zone centrale des bandes fermes une sollicitation en compression verticale et horizontale qui se met en place ou s'accroit en intensité puis se maintient en permanence après 1'exploitation des deux ilots situés de part et d'autre d'une bande ferme.

Le contrôle que nous préconisons consiste donc à mettre en place des mesureurs d'expansion au sein d'une ou deux bandes fermes. Ceux-ci étant disposé au sein de la couche exploitée et horizontalement -avant 1'exploitation des ilots contigüs- doivent mettre en évidence une déformation négative (tassement) résultant de $1^{\prime}$ accroissement de $1 \mathrm{a}$ sollicitation en triple étreinte qui s'exerce sur 1a zone contrôle de la bande ferme pendant et après I'exploitation de ces ilots contigüs.

CHAPITRE III : LES APPLICATIONS ET LES RETOMBEES INDUSTRIELLES DE LA RECHERCHE - LA MINI-TAILLE ILOT

Pour terminer, nous voudrions rappeler un phénomène courant en matière d'innovation I1 s'agit des retombées imprévues à 1'origine de la recherche.

1) au niveau du 1aboratoire et du bureau d'étude, nous possédons désormais grâce au programme VIPLEF et à la mise au point des mesures capables de caractériser les roches rompues, un outil qui nous permettra :

- de calculer un soutènement,

- de calculer la résistance de piliers abandonnés remblayés

- d'aborder la mise au point du calcul des fronts de taille ou de dépilage. 
2) au niveau de l'exploitation minière, 1e fil conducteur qui a permis de réduire les contraintes agissant sur les fronts de dépilage grâce aux îlots réduits (en agissant sur la hauteur des cloches donc sur 1a hauteur des terrains mis en mouvement par le foudroyage) nous a incité à reprendre les essais de taille à soutènement marchant dans les mines de fer de Lorraine.

Tous les essais entrepris avec cette technique d'exploitation avaient conduit à des difficultés qu'on avait pu progressivement éliminer sans pouvoir déboucher sur les pleines possibilités offertes par les tailles et le soutènement marchant. Pour se défendre contre les mises en charge intempestives et brutales capables de détruire les piles pourtant surpuissantes, on avait introduit diverses parades certes efficaces (front de taille très court, piles nombreuses et renforcées, présence d'un rideau de minerai en fond de taille mais qu'il fallait torpiller à $1^{\prime} \operatorname{explosif).~}$

Malheureusement, ces parades introduisaient dans les travaux d'exploitation une gène incompatible avec une productivité suffisante pour rentabiliser les équipements.

Le fait d'introduire la mini-taille (front très court) de $40 \mathrm{~m}$ de 1 ongueur dans les llots réduits de $1 \mathrm{a}$ mine de Serrouville a supprimé tous les inconvénients que nous venons de décrire. La figure 15 donne le plan de la taille introduite pour 1'exploitation de $1^{\prime} \hat{1} 1$ ot réduit et la figure 16 donne 1 a coupe verticale du front de taille montrant une pile et la tarière schématisée du mineur continu.

L'exploitation est conduite par passe de $3,3 \mathrm{~m}$ abattue au mineur continu JEFFREY $120 \mathrm{HR}$.

Les convoyeurs b1indés ont été supprimés. Le mineur continu déverse les produits abattus directement dans des camions de $14 t$ de capacité venant à l'arrière du mineur continu entre les piles et le front. Le soutènement marchant constitué de piles de $800 \mathrm{t}$ (charge de coulissement) se ripe grâce à des poutres reliant les piles 3 par 3 .

Trois hommes desservent le quartier (un conducteur du mineur continu, deux camionneurs). Les rendements abattage atteints industriellement sur un mois se situent à 250 tonnes par homme et par poste $(253,7 \mathrm{t} /$ hp en décembre 1980). Les meilleurs postes ont entraîné des rendements de plus de $300 \mathrm{t}$ par homme et par poste. Il faut souligner gue les camions transportent le minerai sur 500 mètres.

Rappelons qu'il s'agit d'un minerai de densité en place égale à 2,6 et que 1 'ouverture des travaux a $3,5 \mathrm{~m}$ égale à 1 a puissance de la couche. Celle-ci est donc une couche mince pour une mine métallique.

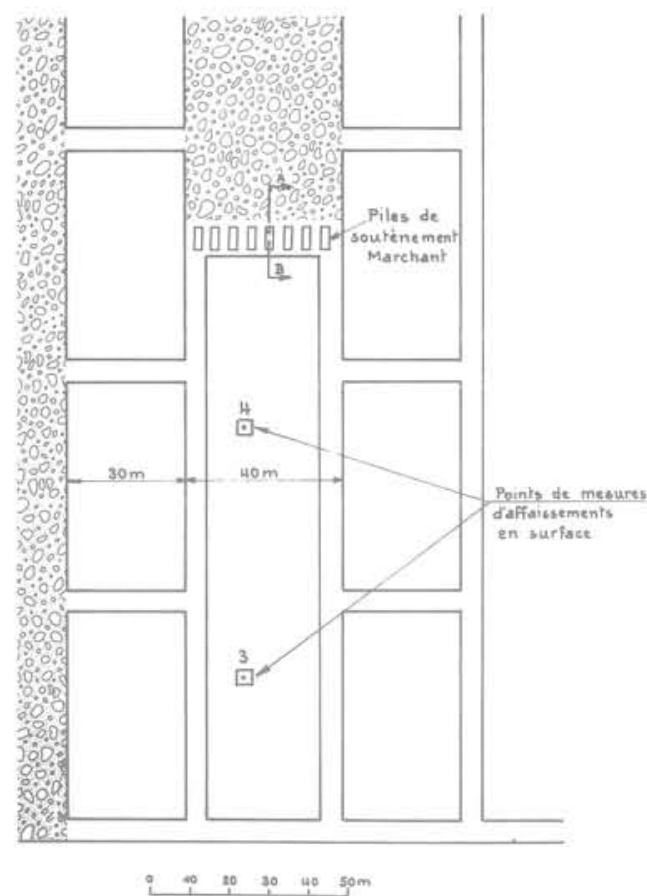

Fig.16 Mini-taille - Ilot Serrouville

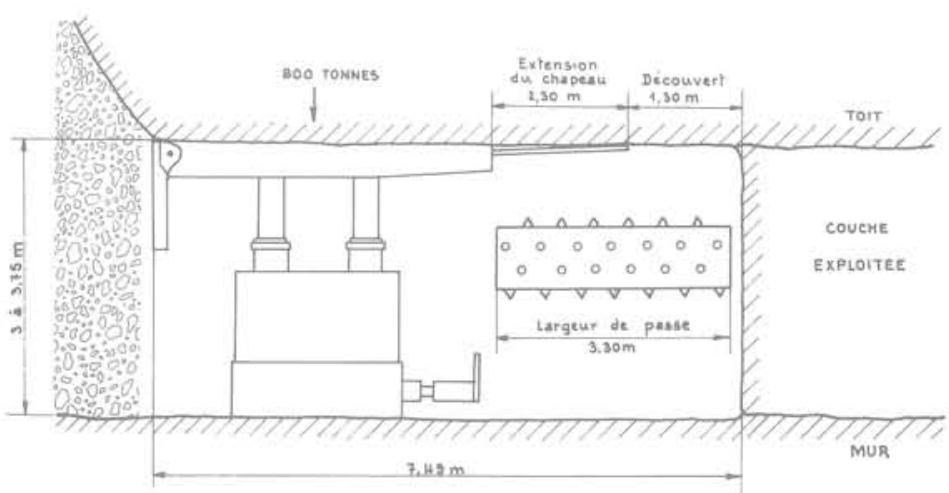

Fig.17 Coupe du front de taille suivant $A B$

De telles nerformances acquises après quelques mois d'essais nous situent dès maintenant au niveau du record mondial.

\section{CHAPITRE IV : CONCLUSIONS}

Cette recherche entreprise pendant quatre ans de 1977 à 1980 a été amplement couronnée de succès. Certes, il reste a améliorer les techniques industrielles et les procédés de calcul. Ceux-ci permettent dès maintenant de sortir de l'empirisme qui était 1a seule ressource du mineur. Les résultats obtenus industriellement sont excellents et 1 aissent présager de nouveaux bonds en avant dans la productivité et la sécurité minières.

Nous voudrions souligner ici un des points importants sur lesque $1 \mathrm{~s}$ nous n'avons pas insisté dans 1e texte. Grâce aux îlots réduits, certes nous avons : 
- diminué les pressions,

- amélioré les rendements et les conditions de travail

- éliminé une grande partie de l'eau faisant irruption dans les travaux du fond,

mais surtout nous avons pu améliorer considérablement le taux de défruitement dans l'ensemble des stots par rapport à celui obtenu avec les piliers abandonnés. Ce gain risque d'être d'autant plus important que $1 \mathrm{a}$ profondeur sera grande. Dans les seules mines de fer de Lorraine, ce taux passe de 45-50\% pour les piliers abandonnés à 60-63\% pour les illots réduits, soit un gain très substantiel.

Différentes techniques faisant appel au savoir-faire du mineur et à la science du mécanicien des roches ont été à l'occasion de cette recherche, mobilisées simultanément :

- in situ pour essayer en vraie grandeur une nouvelle structure minière en partant des données empiriques nées de 1'expérience en particulier de l'expérience des illots classiques.

- in situ pour mesurer les affaissements de surface, les déformations des bandes fermes, les hauteurs des cloches observées et les caractéristiques mécaniques des différents bancs constituant le recouvrement.

- au laboratoire pour comprendre puis modéliser le comportement des matérériaux sollicités au sein de cette nouvelle structure afin de pouvoir en calculer les éléments. Cette importante mobilisation de techniques diverses a permis dans un laps de temps relativement court de mettre au point puis de déterminer les données géométriques et techniques qui président au bon fonctionnement de cette méthode dite des illots.

Mais cette association du mineur et du mécanicien des roches n'est pas suffisante. I1 ne faut pas oublier tout d'abord le personnel des bureaux d'étude qui a conçu, mis au point puis utilisé les appareils de mesure, tout en s'efforçant de synchroniser et de concilier au mieux les travaux d'exploitation et les tâches plus ou moins perturbatrices inhérentes aux mesures. Il ne faut pas oublier non plus le rôle des concepteurs du projet de recherche. I1 s'agit en fait d'imaginer, de lancer et aussi de relancer un projet quelconque ici une nouvelle technique d'exploitation.

Car en ce domaine, comme dans tous les autres, il faut persuader les responsables et les financiers, il faut convaincre le personnel à tous les échelons, il faut soutenir $1^{\prime}$ intérêt de tous, il faut obtenir 1'adhésion de 1'administration qui se doit comme ce fut 1e cas tout au long de cette recherche d'abandonner la lettre du règlement pour n'en garder que l'esprit. Oue tous ici soient remerciés. Nous voudrions direenfin combien ont été efficaces les aides financières apportées :

- par la DGRST, pour la partie laboratoire et recherche scientifique

- par la CECA pour les essais in situ

- par 1a profession minière qui a poursuivi une politique audacieuse d'innovation malgré une situation économique catastrophique. 